TRANSACTIONS OF THE

AMERICAN MATHEMATICAL SOCIETY

Volume 353, Number 6, Pages 2245-2274

S 0002-9947(00)02691-X

Article electronically published on November 28, 2000

\title{
NON-SPECIAL, NON-CANAL ISOTHERMIC TORI WITH SPHERICAL LINES OF CURVATURE
}

\author{
HOLLY BERNSTEIN
}

\begin{abstract}
This article examines isothermic surfaces smoothly immersed in Möbius space. It finds explicit examples of non-special, non-canal isothermic tori with spherical lines of curvature in two systems by analyzing Darboux transforms of Dupin tori. In addition, it characterizes the property of spherical lines of curvature in terms of differential equations on the Calapso potential of the isothermic immersion, and investigates the effect of classical transformations on this property.
\end{abstract}

\section{INTRODUCTION}

Let $f: S \rightarrow(M, g)$ be a smooth immersion of a surface $S$ into a 3-dimensional, oriented, Riemannian manifold $M$. In terms of local coordinates $(x, y)$ on $S$, the first and second fundamental forms are given by

$$
\begin{aligned}
I & =E d x^{2}+2 F d x d y+G d y^{2}, \\
I I & =l d x^{2}+2 m d x d y+n d y^{2} .
\end{aligned}
$$

Local coordinates for which $E=G$ and $F=0$ are called isothermal. Local coordinates for which $m=0$ are called principal. The immersion $f$ is isothermic if for any point $p \in S$, there exists a neighborhood with local coordinates that are both principal and isothermal. Examples of isothermic immersions include surfaces of revolution, cones, cylinders, and constant mean curvature immersions.

The local properties of isothermic immersions were studied extensively in the late 1800 's by geometers including Bianchi and Darboux. In recent years, interest in isothermic immersions has reappeared in connection with Bonnet's problem. Two non-congruent isometric immersions of a surface into $\mathbb{R}^{3}$ that have the same mean curvature function are called Bonnet mates. Bonnet's problem asks if there exist a pair of compact embedded surfaces of given genus that are Bonnet mates. Tribuzy proved that a compact embedded surface of genus zero does not admit a Bonnet mate [Tri80]. Moreover, with Lawson, Tribuzy proved that a compact embedded surface with non-constant mean curvature admits at most one Bonnet mate [LT81]. For genus greater than zero, however, existence remains an open question.

Kamberov, Pedit, and Pinkall have shown that locally each pair of Bonnet mates arises from an isothermic immersion KPP98. Thus, if there exist two compact embedded tori that solve Bonnet's problem, then these tori might arise from an

Received by the editors August 15, 1999 and, in revised form, March 10, 2000.

1991 Mathematics Subject Classification. Primary 53A05; Secondary 51B10, 58 G37.

Key words and phrases. Isothermic, tori, Darboux transform, Möbius geometry, moving frames, umbilic loci. 
isothermic torus. This makes isothermic immersions in general, and isothermic tori in particular, an interesting area of current research. In contrast to the classical investigations, this research focuses on global results.

Prior to this article, the known examples of isothermic tori were either tori of revolution or constant mean curvature tori immersed in one of $\mathbb{R}^{3}, S^{3}$, or $\mathbb{H}^{3}$. The latter were discovered by U. Pinkall, I. Sterling, H. Wente, R. Walter, U. Abresch, and A. Bobenko [PS89, Wen86, Wal87, Wal89, Abr87, Bob91]. However, none of these tori appear to be helpful in solving Bonnet's problem. The property of being isothermic is preserved under the action of the group of conformal transformations, also called the group of Möbius transformations. Thus, trivial new examples of isothermic tori can be generated by taking Möbius transformations of known examples. A torus that is Möbius equivalent to a torus of revolution is called canal, while a torus that is Möbius equivalent to a constant mean curvature torus in one of the three space forms is called special.

This article finds explicit examples of isothermic tori that are not Möbius transformations of the known examples. In addition to proving the existence of nonspecial, non-canal isothermic tori, these tori exhibit several interesting properties: They arise from the Darboux transforms of a standard torus of revolution, and their parametrizations are given in terms of the trigonometric functions. These tori have spherical lines of curvature in two systems. They belong to a countable number of conformal classes, and in each such class, there are uncountably many tori that are not Möbius equivalent. Some of these tori are umbilic free, some have isolated umbilic points, and some have curves of umbilic points that are distinct from the coordinate curvature lines. It was thought that isothermic tori with such curves of umbilics exist, but to our knowledge, the tori in this article provide the first explicit examples.

Since some of the background information used in this endeavor is difficult to obtain, Section 2 contains extensive background material. Section 3 characterizes the property of spherical lines of curvature in terms of differential equations on the Calapso potential of the immersion, and investigates how certain transformations of isothermic immersions affect the property of spherical curvature lines. Section 4 demonstrates the existence of non-special, non-canal isothermic tori with spherical lines of curvature by finding explicit examples. It also explores the properties of these tori. Section 5 contains examples and pictures.

Special thanks go to Gary Jensen, Emilio Musso, and George Kamberov for many helpful discussions.

\section{BACKGROUND}

2.1. Möbius geometry. We use the model of Möbius space used by Bryant and Musso Bry84, CM98]. After developing the basic setup, we show how the three space forms are embedded in Möbius space. We explore how the space of oriented spheres appears in Möbius space. Finally, we examine the method of moving frames in Möbius space as it applies to curves and surfaces.

2.1.1. Möbius space and the Möbius group. Let $\mathbb{L}^{5}$ be the vector space $\mathbb{R}^{5}$ with the inner product

$$
\langle x, y\rangle=-\left(x^{0} y^{4}+x^{4} y^{0}\right)+x^{1} y^{1}+x^{2} y^{2}+x^{3} y^{3}=g_{i j} x^{i} y^{j} .
$$


Orientation is defined by

$$
\Omega=d x^{0} \wedge d x^{1} \wedge d x^{2} \wedge d x^{3} \wedge d x^{4}>0 .
$$

Time orientation is defined by the positive light cone

$$
\mathcal{L}^{+}=\left\{x \in \mathbb{L}^{5}:\langle x, x\rangle=0, x^{0}+x^{4}>0\right\} .
$$

Definition 2.1. The Möbius group $G$ is the identity component of the pseudoorthogonal group of (11).

Let $\left(\epsilon_{0}, \epsilon_{1}, \epsilon_{2}, \epsilon_{3}, \epsilon_{4}\right)$ be the standard basis of $\mathbb{R}^{5}$, and let $A \in G$. Let $A_{i}=A \epsilon_{i} \in$ $\mathbb{L}^{5}$ denote the column vectors of $A$. Then $\left(A_{0}, A_{1}, A_{2}, A_{3}, A_{4}\right)$ is a basis for $\mathbb{L}^{5}$ such that

$$
\left\langle A_{i}, A_{j}\right\rangle=g_{i j}, \quad A_{0}, A_{4} \in \mathcal{L}^{+}, \quad \Omega\left(A_{0}, A_{1}, A_{2}, A_{3}, A_{4}\right)=\operatorname{det} A=1 .
$$

Regard the $A_{i}$ as $\mathbb{L}^{5}$ valued functions on $G$. Since the $A_{i}$ form a basis of $\mathbb{L}^{5}$, there exist unique left invariant 1 -forms $\omega_{j}^{i}$ such that

$$
d A_{i}=\omega_{i}^{j} A_{j} .
$$

Differentiating (2), we obtain the Cartan structure equations

$$
d \omega_{j}^{i}=-\omega_{k}^{i} \wedge \omega_{j}^{k}
$$

Differentiating the relationship $\left\langle A_{i}, A_{j}\right\rangle=g_{i j}$ gives the symmetry equations

$$
\omega_{i}^{k} g_{k j}+\omega_{j}^{k} g_{i k}=0
$$

Definition 2.2. Möbius space is the real hyperquadric in $\mathbb{R P}^{4}$ defined by

$$
\mathcal{M}=\left\{[y] \in \mathbb{R P}^{4}:\langle y, y\rangle=0\right\} .
$$

The unit sphere $S^{3}$ in $\mathbb{R}^{4}$ is diffeomorphic to the space $\mathcal{M}$ via the map

$$
{ }^{t}\left(x^{0}, x^{1}, x^{2}, x^{3}\right) \in S^{3} \mapsto \quad\left[\frac{1+x^{0}}{2}, \frac{x^{1}}{\sqrt{2}}, \frac{x^{2}}{\sqrt{2}}, \frac{x^{3}}{\sqrt{2}}, \frac{1-x^{0}}{2}\right] \in \mathcal{M} .
$$

The Möbius group acts transitively on $\mathcal{M}$ on the left via $A[x]=[A x]$, and the projection map

$$
\pi: A \in G \rightarrow\left[A_{0}\right] \in \mathcal{M}
$$

makes $G$ into a principal fiber bundle with structure group

$$
G_{0}=\left\{\left[\begin{array}{ccc}
r^{-1} & { }^{t} p X & \frac{1}{2} r^{t} p p \\
0 & X & r p \\
0 & 0 & r
\end{array}\right]: r>0, X \in S O(3), p \in \mathbb{R}^{3}\right\} .
$$

The forms $\omega_{0}^{1}, \omega_{0}^{2}, \omega_{0}^{3}$ generate the space of semi-basic 1-forms. Letting $A(r, X, p) \in$ $G_{0}$, we see that

$$
\begin{gathered}
R_{A}^{*}\left(\omega_{0}^{1} \wedge \omega_{0}^{2} \wedge \omega_{0}^{3}\right)=\frac{1}{r^{3}} \omega_{0}^{1} \wedge \omega_{0}^{2} \wedge \omega_{0}^{3}, \\
R_{A}^{*}\left(\left(\omega_{0}^{1}\right)^{2}+\left(\omega_{0}^{2}\right)^{2}+\left(\omega_{0}^{3}\right)^{2}\right)=\frac{1}{r^{2}}\left(\left(\omega_{0}^{1}\right)^{2}+\left(\omega_{0}^{2}\right)^{2}+\left(\omega_{0}^{3}\right)^{2}\right) .
\end{gathered}
$$

Thus $\omega_{0}^{1} \wedge \omega_{0}^{2} \wedge \omega_{0}^{3}$ and $\left(\omega_{0}^{1}\right)^{2}+\left(\omega_{0}^{2}\right)^{2}+\left(\omega_{0}^{3}\right)^{2}$ are well defined on $\mathcal{M}$ up to a positive multiple. This implies that these forms induce an orientation and a conformal structure, respectively, on $\mathcal{M}$. 
2.1.2. Space forms in Möbius space. Let $c \in\{-1,0,1\}$, and let $M_{c}$ denote the 3dimensional space form of constant sectional curvature $c$; that is, the space $M_{0}$ is the Euclidean space $x^{0}=1$ in $\mathbb{R}^{4}$, the space $M_{1}$ is the unit 3 -sphere in $\mathbb{R}^{4}$, and the space $M_{(-1)}$ is the hyperbolic space of curvature -1 defined by

$$
\mathbb{H}^{3}=\left\{x \in \mathbb{R}^{4}:-\left(x^{0}\right)^{2}+\left(x^{1}\right)^{2}+\left(x^{2}\right)^{2}+\left(x^{3}\right)^{2}=-1, x^{0} \geq 1\right\} .
$$

We freely identify the point ${ }^{t}\left(1, x^{1}, x^{2}, x^{3}\right) \in M_{0}$ with the point ${ }^{t}\left(x^{1}, x^{2}, x^{3}\right) \in \mathbb{R}^{3}$. Conformal embeddings of $M_{c}$ into Möbius space are given by

$$
\begin{aligned}
r_{1}:{ }^{t}\left(x^{0}, x^{1}, x^{2}, x^{3}\right) \in M_{1} & \mapsto{ }^{t}\left[\frac{1+x^{0}}{2}, \frac{x^{1}}{\sqrt{2}}, \frac{x^{2}}{\sqrt{2}}, \frac{x^{3}}{\sqrt{2}}, \frac{1-x^{0}}{2}\right], \\
r_{(-1)}:{ }^{t}\left(x^{0}, x^{1}, x^{2}, x^{3}\right) \in M_{(-1)} & \mapsto{ }^{t}\left[\frac{1+x^{0}}{2}, \frac{x^{1}}{\sqrt{2}}, \frac{x^{2}}{\sqrt{2}}, \frac{x^{3}}{\sqrt{2}}, \frac{x^{0}-1}{2}\right], \\
r_{0}:{ }^{t}\left(1, x^{1}, x^{2}, x^{3}\right) \in M_{0} & \mapsto{ }^{t}\left[1, \frac{x^{1}}{\sqrt{2}}, \frac{x^{2}}{\sqrt{2}}, \frac{x^{3}}{\sqrt{2}}, \frac{|x|^{2}}{4}\right] .
\end{aligned}
$$

As remarked in Section 2.1.1, the map $r_{1}$ takes $S^{3}$ diffeomorphically onto $\mathcal{M}$. Identifying $\mathcal{M}$ with $S^{3}$, the map $r_{(-1)}$ takes $\mathbb{H}^{3}$ diffeomorphically onto the "northern" hemisphere of $\mathcal{M}$, and the map $r_{0}$ is an inverse stereographic projection from the south pole. This stereographic projection is given by

$$
r_{0}^{-1}:{ }^{t}\left[y^{0}, y^{1}, y^{2}, y^{3}, y^{4}\right] \mapsto \sqrt{2}^{t}\left(\frac{y^{1}}{y^{0}}, \frac{y^{2}}{y^{0}}, \frac{y^{3}}{y^{0}}\right) .
$$

In particular, the point ${ }^{t}[0,0,0,0,1] \in \mathcal{M}=S^{3}$ corresponds to the point at infinity in $\mathbb{R}^{3}$. We denote this point by $P_{\infty}$.

Let $H_{c}$ be the group of orientation preserving isometries of $M_{c}$; that is,

$$
H_{0}=\mathbb{E}(3), \quad H_{1}=S O(4), \quad H_{-1}=S O(3,1) .
$$

Recall that $H_{c}$ is a principal fiber bundle over $M_{c}$ via the map $\pi_{c}: C \rightarrow C_{0}$, which projects the matrix $C$ onto its first column vector. Let $\rho_{c}: H_{c} \rightarrow G$ denote the faithful representations defined by

$$
\rho_{ \pm 1}(C)=\left[\begin{array}{ccc}
\frac{1+C_{0}^{0}}{2} & \frac{C_{j}^{0}}{\sqrt{2}} & \frac{ \pm\left(1-C_{0}^{0}\right)}{2} \\
\frac{C_{0}^{i}}{\sqrt{2}} & C_{j}^{i} & \frac{\mp C_{0}^{i}}{\sqrt{2}} \\
\frac{ \pm\left(1-C_{0}^{0}\right)}{2} & \frac{\mp C_{j}^{0}}{\sqrt{2}} & \frac{1+C_{0}^{0}}{2}
\end{array}\right] \text {, and } \rho_{0}(C)=\left[\begin{array}{ccc}
1 & 0 & 0 \\
\frac{C_{0}^{i}}{\sqrt{2}} & C_{j}^{i} & 0 \\
\frac{\left|C_{0}\right|^{2}}{4} & \frac{C_{0} \cdot C_{i}}{\sqrt{2}} & 1
\end{array}\right]
$$

The diagram

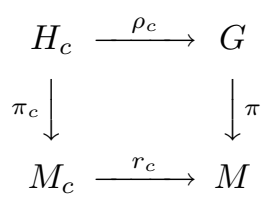

commutes, and it makes $\rho_{c}$ into an injective homomorphism of principal fiber bundles; that is,

$$
r_{c}(C \cdot x)=\rho_{c}(C) \cdot r_{c}(x),
$$

for all $C \in H_{c}$ and $x \in M_{c}$. Thus $\pi_{c}: H_{c} \rightarrow M_{c}$ may be viewed as a reduced subbundle of $\pi: G \rightarrow \mathcal{M}$ on the image of $M_{c}$ in $\mathcal{M}$. 
2.1.3. The Möbius group as orientation preserving conformal transformations of $\mathbb{R}^{3}$. Let $A \in G$ and $x \in \mathbb{R}^{3}$. The Möbius group $G$ acts on $\mathbb{R}^{3}$ via

$$
L_{A}(x):=r_{0}^{-1}\left(A \cdot r_{0}(x)\right) \text {. }
$$

Via this action the Möbius group can be viewed as the pseudogroup of orientation preserving conformal transformations of Euclidean space [Nic96].

2.1.4. Surface theory in Möbius space. In this section we apply the method of moving frames to a surface immersed in Möbius space. The details of this frame reduction in Möbius space are well developed by Bryant in Bry84, so we only give a summary of its most important features below.

Assumption 2.1. Throughout this article, all maps are assumed to be smooth unless otherwise noted.

Definition 2.3. Let $S$ be a Riemann surface, let $U$ be an open subset of $S$, and let $f: S \rightarrow \mathcal{M}$ be a conformal immersion. A Möbius frame field along $f$ is a map $B: U \rightarrow G$ such that $f(x)=\left[B_{0}(x)\right]$ for all $x \in U$.

Given a Möbius frame field $B$ along $f$, consider the pullback of the MaurerCartan form $\omega=\left(\omega_{j}^{i}\right)$ of $G$ to $S$ :

$$
\left(\beta_{j}^{i}\right)=\beta=B^{-1} d B=B^{*} \omega
$$

Any other Möbius frame field on $U$ is given by

$$
\tilde{B}=B g,
$$

where $g: U \rightarrow G_{0}$. Under this change of frame, the pullback of the Maurer-Cartan form transforms as follows:

$$
\tilde{B}^{*}(\omega)=(B g)^{-1} d(B g)=g^{-1} \beta g+g^{-1} d g .
$$

Definition 2.4. A first order frame field is a frame field for which

$$
\beta_{0}^{3}=0, \quad \beta_{0}^{1} \wedge \beta_{0}^{2}>0 .
$$

First order frame fields exist in a neighborhood of any point.

Given a first order frame field, differentiating $\beta_{0}^{3}=0$ and applying Cartan's Lemma implies $\beta_{i}^{3}=h_{i j} \beta_{0}^{j}$, where $h_{i j}=h_{j i}$. The form

$$
\tilde{\Psi}=\left(\frac{1}{4}\left(h_{11}-h_{22}\right)^{2}+h_{12}^{2}\right) \beta_{0}^{1} \wedge \beta_{0}^{2}
$$

is invariant under a change of first order frame field. In other words, $\tilde{\Psi}$ induces a global, non-negative 2 -form $\Psi$ on $S$, given by equation (4) with respect to any first order frame field.

Definition 2.5. The umbilic locus of $f$ is the set $\left\{p \in S: \Psi_{p}=0\right\}$. Elements of the umbilic locus are called umbilic points.

If $f: S \rightarrow M_{c}$ is an immersion, then consider the map $r_{c} \circ f$. A point of $S$ is in the umbilic locus of $r_{c} \circ f$ if and only if it is an umbilic point for $f$ in the classical sense.

The umbilic locus of the surface is a conformal invariant. It is a closed set. On the complement of the umbilic locus, two further frame reductions can be made. The end result is the following: 
Definition 2.6. A Möbius frame field $B: U \rightarrow G$ along a conformal immersion $f: S \rightarrow \mathcal{M}$ is a normal frame field if

$$
\beta_{0}^{1} \wedge \beta_{0}^{2}>0, \quad \beta_{0}^{3}=0=\beta_{3}^{0}, \quad \beta_{1}^{3}=\beta_{0}^{1}, \quad \beta_{2}^{3}=-\beta_{0}^{2} .
$$

Theorem 2.1 ([Bry84] $)$. Let $f: S \rightarrow \mathcal{M}$ be a conformal immersion. A normal frame field exists in a neighborhood of any non-umbilic point of $S$.

If $B: U \rightarrow G$ is a normal frame field, then any other normal frame field on $U$ is given by $\left(B_{0}, \epsilon B_{1}, \epsilon B_{2}, B_{3}, B_{4}\right)$, where $\epsilon= \pm 1$. Let

$$
P_{n}(f)=\{(x, B(x)): x \in S, B: U \ni x \rightarrow G \text { is a normal frame }\} .
$$

The projection $\pi: P_{n}(f) \rightarrow S$ defines the bundle of normal frames. This bundle is a $\mathbb{Z}_{2}$ principal bundle. The frame reduction is now complete, because a change of normal frame does not lead to any advantageous simplifications of the MaurerCartan form.

Differentiating the conditions (5) and applying Cartan's Lemma implies that there exist smooth functions $p_{1}, p_{2}, p_{3}, q_{1}, q_{2}: U \rightarrow \mathbb{R}$ such that the Maurer-Cartan form of the normal frame $B$ takes the form

$$
\left(\begin{array}{ccccc}
-2 q_{2} \beta_{0}^{1}+2 q_{1} \beta_{0}^{2} & p_{1} \beta_{0}^{1}+p_{2} \beta_{0}^{2} & -p_{2} \beta_{0}^{1}+p_{3} \beta_{0}^{2} & 0 & 0 \\
\beta_{0}^{1} & 0 & -q_{1} \beta_{0}^{1}-q_{2} \beta_{0}^{2} & -\beta_{0}^{1} & p_{1} \beta_{0}^{1}+p_{2} \beta_{0}^{2} \\
\beta_{0}^{2} & q_{1} \beta_{0}^{1}+q_{2} \beta_{0}^{2} & 0 & \beta_{0}^{2} & -p_{2} \beta_{0}^{1}+p_{3} \beta_{0}^{2} \\
0 & \beta_{0}^{1} & -\beta_{0}^{2} & 0 & 0 \\
0 & \beta_{0}^{1} & \beta_{0}^{2} & 0 & 2 q_{2} \beta_{0}^{1}-2 q_{1} \beta_{0}^{2}
\end{array}\right) .
$$

The functions $p_{1}, p_{2}, p_{3}$ are constant along the fibers of $\pi_{n}$. Thus, there are well defined global functions $J, W, M: S \rightarrow \mathbb{R}$ such that locally

$$
W=\frac{1}{2}\left(p_{1}-p_{3}\right), \quad J=\frac{1}{2}\left(p_{1}+p_{3}\right), \quad M=p_{2} .
$$

Bryant proved that an umbilic free immersion for which $W \equiv 0$ is a Willmore immersion Bry84. An immersion for which $J \equiv 0$ is called an immersion of spherical type. Such immersions are Möbius equivalent to a minimal immersion into one of the space forms CM98. We will see that an immersion for which $M \equiv 0$ is isothermic [Mus97].

Pulling the structure equations (3) back to the surface $S$ yields the structure equations of the immersion:

$$
\begin{aligned}
d \beta_{0}^{1} & =-q_{1} \beta_{0}^{1} \wedge \beta_{0}^{2}, \\
d \beta_{0}^{2} & =-q_{2} \beta_{0}^{1} \wedge \beta_{0}^{2}, \\
d q_{1} \wedge \beta_{0}^{1}+d q_{2} \wedge \beta_{0}^{2} & =\left(1+p_{1}+p_{3}+q_{1}^{2}+q_{2}^{2}\right) \beta_{0}^{1} \wedge \beta_{0}^{2}, \\
d q_{2} \wedge \beta_{0}^{1}-d q_{1} \wedge \beta_{0}^{2} & =-p_{2} \beta_{0}^{1} \wedge \beta_{0}^{2}, \\
d p_{1} \wedge \beta_{0}^{1}+d p_{2} \wedge \beta_{0}^{2} & =\left(4 q_{2} p_{2}+q_{1}\left(3 p_{1}+p_{3}\right)\right) \beta_{0}^{1} \wedge \beta_{0}^{2}, \\
d p_{2} \wedge \beta_{0}^{1}-d p_{3} \wedge \beta_{0}^{2} & =\left(4 q_{1} p_{2}-q_{2}\left(p_{1}+3 p_{3}\right)\right) \beta_{0}^{1} \wedge \beta_{0}^{2} .
\end{aligned}
$$

2.1.5. Oriented spheres in Möbius space. Recall that under the action of the conformal transformations, planes in $\mathbb{R}^{3}$ are special cases of spheres in $\mathbb{R}^{3}$. Thus, the space of oriented spheres in $\mathbb{R}^{3}$ is the space consisting of all oriented spheres in $\mathbb{R}^{3}$ along with all oriented planes in $\mathbb{R}^{3}$. Via stereographic projection, this space may also be viewed as the space of oriented spheres in $S^{3}=\mathcal{M}$. One of the most useful 
features of Möbius geometry is the identification of the space of oriented spheres with the quadric

$$
\mathfrak{S}=\left\{B \in \mathbb{L}^{5}:\langle B, B\rangle=1\right\} .
$$

The oriented sphere $\sigma(p, r) \subset \mathbb{R}^{3}$ with center $p \in \mathbb{R}^{3}$ and signed radius $r \in \mathbb{R}^{*}$ is identified with the point

$$
s(p, r)={ }^{t}\left(\frac{\sqrt{2}}{r}, \frac{p^{1}}{r}, \frac{p^{2}}{r}, \frac{p^{3}}{r}, \frac{|p|^{2}-r^{2}}{2 \sqrt{2} r}\right) \in \mathfrak{S} .
$$

Here a positive radius denotes the orientation given by the inward normal; negative, the outward normal.

The oriented plane $\pi(n, h)$ with normal $n \in S^{2}$ and equation $n \cdot v=h$ is identified with the point

$$
p(n, h)={ }^{t}\left(0, n^{1}, n^{2}, n^{3}, \frac{h}{\sqrt{2}}\right) \in \mathfrak{S} .
$$

Conversely, if $B \in \mathfrak{S}$, let

$$
B^{\perp}=\left\{y \in \mathbb{L}^{5}:\langle y, B\rangle=0\right\} .
$$

The stereographic projection of $B^{\perp} \cap \mathcal{M}$ into $\mathbb{R}^{3}$ will be an oriented sphere in $\mathbb{R}^{3}$ if $b^{0} \neq 0$, and an oriented plane in $\mathbb{R}^{3}$ if $b^{0}=0$.

Definition 2.7. Two spheres are in oriented contact if they are tangent and induce the same orientation on their common tangent plane. They are in oppositely oriented contact if they are tangent and induce opposite orientations on their common tangent plane.

Remark 2.1. The relative positions of two oriented spheres $B_{1}, B_{2} \in \mathfrak{S}$ may be determined as follows Nic96:

1. If $0 \leq\left|\left\langle B_{1}, B_{2}\right\rangle\right|<1$, then the spheres (planes) corresponding to $B_{1}$ and $B_{2}$ intersect along a circle (line), and $\left\langle B_{1}, B_{2}\right\rangle=0$ if and only if the two spheres intersect orthogonally.

2. The two spheres are in oriented contact if and only if $\left\langle B_{1}, B_{2}\right\rangle=1$, and the two spheres are in oppositely oriented contact if and only if $\left\langle B_{1}, B_{2}\right\rangle=-1$.

3. The two spheres are disjoint if and only if $\left|\left\langle B_{1}, B_{2}\right\rangle\right|>1$.

Remark 2.2. If $A \in G$, the vectors $A_{1}, A_{2}, A_{3} \in \mathfrak{S}$ are oriented spheres that intersect each other orthogonally. The points $\left[A_{0}\right],\left[A_{4}\right] \in \mathcal{M}$ are the common intersection points of these three spheres.

Remark 2.3. Let $S$ be a Riemann surface, and let $f: S \rightarrow \mathcal{M}$ be a conformal immersion. By doing a Möbius transformation, we may assume without loss of generality that $P_{\infty} \notin f(S)$, so that the map $\tilde{f}=r_{0}^{-1} \circ f$ is an immersion into $\mathbb{R}^{3}$. Take any first order frame $(\tilde{f}, e)$ along $\tilde{f}$ around a point $p \in S$. The Euclidean immersion $\tilde{f}$ has oriented affine tangent plane $\tilde{f}(p)+\left.d \tilde{f}\right|_{p}\left(T_{p} S\right)$ at the point $\tilde{f}(p)$. This plane has unit normal vector $e_{3}(p)$. Thus the oriented affine tangent plane to $\tilde{f}(S)$ at $\tilde{f}(p)$ corresponds to the point $\left(0, e_{3}, \frac{e_{3} \cdot \tilde{f}}{\sqrt{2}}\right)(p) \in \mathfrak{S}$. Any sphere or plane in oriented contact with $\tilde{f}$ at $p$ is in oriented contact with this plane. 
Definition 2.8. A sphere congruence is a map $s: S \rightarrow \mathfrak{S}$. Let $f: S \rightarrow \mathcal{M}$ be a conformal immersion, and let $\tilde{f}=r_{0}^{-1} \circ f: S \rightarrow \mathbb{R}^{3}$ be the corresponding immersion into Euclidean space. The immersion $(S, f)$ is an envelope of the sphere congruence $s: S \rightarrow \mathfrak{S}$ if for every $p \in S$, the oriented affine tangent plane $\tilde{f}(p)+\left.d \tilde{f}\right|_{p}\left(T_{p} S\right)$ and $s(p)$ are in oriented contact at $\tilde{f}(p)$.

Remark 2.4. With notation as in Remark 2.3, let $B=\rho_{0}((\tilde{f}, e))$ be the projection of the frame $(\tilde{f}, e)$ into $G$. Then $B$ is a first order Möbius frame field along $f$. Any other first order Möbius frame field $A$ along $f$ has fourth column vector of the form $A_{3}=q B_{0}+B_{3}$, where $q$ is a smooth real valued function. Thus $\left\langle A_{3}, B_{3}\right\rangle=1$, which proves that $B_{3}$ and $A_{3}$ are in oriented contact. Since $B_{3}$ is the oriented affine tangent plane of $\tilde{f}$, this shows that if $A$ is a first order Möbius frame along $f$, then $f$ is an envelope of $A_{3}$.

Finally, suppose that $c \in\{-1,1\}$. A sphere in $M_{c}$ is the intersection of $M_{c} \subset \mathbb{R}^{4}$ with a hyperplane in $\mathbb{R}^{4}$ Wal89. If $B \in \mathfrak{S}$, then the equation defining $B^{\perp} \cap r_{c}\left(M_{c}\right)$, namely $\left\langle B, r_{c}(z)\right\rangle=0$, is precisely the equation of a hyperplane in $\mathbb{R}^{4}$. Conversely, any hyperplane intersecting $M_{c}$ can be represented by an element of $\mathfrak{S}$. Thus $\mathfrak{S}$ is also the space of spheres in hyperbolic and spherical geometry.

2.1.6. Curve theory in Möbius space. The method of moving frames can also be applied to curves immersed in Möbius space. This theory was developed by R. Sulanke [Sul81. He demonstrated that there are four frame reductions for a noncircular curve immersed in $\mathcal{M}=S^{3}$. In our work, we only need third order frames, so we only summarize the reduction up to this point.

Let $f: I \rightarrow \mathcal{M}$ be an immersed curve. Let $b=\left(b_{0}, b_{1}, b_{2}, b_{3}, b_{4}\right)$ be a Möbius frame along $f$; that is, $f(t)=\left[b_{0}(t)\right]$. Let $\left(\beta_{j}^{i}\right)=b^{-1} d b$ be the pullback of the Maurer-Cartan form of the Möbius group to $I$.

Definition 2.9. A first order frame field along $f$ is a frame for which

$$
\beta_{0}^{2}=0=\beta_{0}^{3} \text {. }
$$

Definition 2.10. A second order frame field along $f$ is a first order frame for which

$$
\beta_{1}^{2}=0=\beta_{1}^{3} .
$$

Definition 2.11. Let $b: I \rightarrow G$ be a second order frame field along $f$. If

$$
\beta_{2}^{0}=0=\beta_{3}^{0},
$$

then the curve is called type (1). Otherwise the curve is called type (2).

Theorem 2.2 ([Sul81] ). The curve $f$ is of type (1) if and only if $f(I)$ is contained in a circle in $\mathcal{M}=S^{3}$.

Definition 2.12. Let $f: I \rightarrow \mathcal{M}$ be a curve of type (2). A third order frame field along $f$ is a second order frame for which

$$
\beta_{2}^{0}=\beta_{0}^{1}, \quad \beta_{3}^{0}=0 .
$$

The forms $\beta_{0}^{1}, \beta_{2}^{3}$ are third order invariants. 
After the third reduction, the Maurer-Cartan form of a frame field along a curve of type (2) has taken the form

$$
\beta=\left(\begin{array}{ccccc}
\beta_{0}^{0} & \beta_{1}^{0} & \beta_{0}^{1} & 0 & 0 \\
\beta_{0}^{1} & 0 & 0 & 0 & \beta_{1}^{0} \\
0 & 0 & 0 & -\beta_{2}^{3} & \beta_{0}^{1} \\
0 & 0 & \beta_{2}^{3} & 0 & 0 \\
0 & \beta_{0}^{1} & 0 & 0 & -\beta_{0}^{0}
\end{array}\right) .
$$

Notice that the vector $b_{3}$ is constant if and only if $\beta_{2}^{3}=0$.

The next lemma follows naturally from Sulanke's research:

Lemma 2.1. Let $f: I \rightarrow \mathcal{M}$ be an immersed curve of type (2). Let $b: I \rightarrow G$ be a third order frame field along $f$. Then $f(I)$ lies in a sphere in $\mathcal{M}$ if and only if $\beta_{2}^{3}=0$; in particular, $f(I)$ lies in the sphere $b_{3}$.

2.2. Isothermic immersions. Let the map $f: S \rightarrow(M, g)$ be a conformal immersion into a 3 -dimensional, oriented Riemannian manifold $(M, g)$.

Definition 2.13. Local coordinates $(x, y)$ that diagonalize the first fundamental form are called isothermal; that is,

$$
I=e^{2 \phi}\left(d x^{2}+d y^{2}\right) .
$$

Notice that with respect to isothermal coordinates, the first fundamental form is conformal to the standard metric on $\mathbb{R}^{2}$.

Definition 2.14. Local coordinates $(x, y)$ for which the parameter curves are lines of curvature are called principal.

Definition 2.15. The map $f$ is isothermic if at any point $p \in S$, there exist local coordinates that are both principal and isothermal; that is, at any point, there are local coordinates $(x, y)$ such that

$$
\begin{gathered}
I=e^{2 \phi}\left(d x^{2}+d y^{2}\right), \\
I I=e^{2 \phi}\left(a d x^{2}+c d y^{2}\right) .
\end{gathered}
$$

Here $a$ and $c$ are the principal curvatures of the immersion.

Example 2.1. Examples of isothermic immersions include surfaces of revolution, cones, and cylinders in $\mathbb{R}^{3}$, as well as constant mean curvature immersions in space forms.

There are two main difficulties with the classical definition of isothermic. First, it is not coordinate free. Second, it often fails to define the notion of isothermic at an umbilic point. There are several suggestions for a coordinate free definition of isothermic. We present one, due to E. Musso, below [Mus97]:

Definition 2.16. A polarization on a Riemann surface $S$ is a holomorphic quadratic differential $\mathcal{Q}$ on $S$ that is not identically zero. The surface $(S, \mathcal{Q})$ is called a polarized surface. An isothermic chart is a complex parameter $z: U \subseteq S \rightarrow \mathbb{C}$ such that $\left.\mathcal{Q}\right|_{U}=d z d z$. Isothermic charts exist near any point $p \in S$ such that $\left.\mathcal{Q}\right|_{p} \neq 0$.

Note that if $z: U \rightarrow \mathbb{C}$ is an isothermic chart, then any other such chart on $U$ is given by $\tilde{z}= \pm z+b$, where $b \in \mathbb{C}$ is locally constant. 
Definition 2.17. Let $S$ be a Riemann surface, and let $f: S \rightarrow(M, g)$ be a conformal immersion of $S$ into a 3-dimensional, oriented Riemannian manifold $(M, g)$. Let $I I^{(2,0)}$ be the Hopf differential of $f$. The immersion $f$ is called isothermic if there exist a polarization $\mathcal{Q}$ of $S$ and a real-valued smooth function $m: S \rightarrow \mathbb{R}$ such that $I I^{(2,0)}=m \mathcal{Q}$.

Write $\mathcal{Q} \overline{\mathcal{Q}}=\lambda^{4} I^{2}$, where $\lambda: S \rightarrow[0, \infty)$. If the polarization is nowhere zero, then $\lambda$ is smooth. At zeros of the polarization, the function $\lambda$ is continuous, but not differentiable. The Calapso potential of $f$ is the map $\Phi: S \rightarrow \mathbb{R}$ defined by $\Phi=2 m \lambda$.

Remark 2.5. Let $f: S \rightarrow M_{c}$ be a constant mean curvature immersion. Recall that the metric induced on $S$ by $f$ gives $S$ a complex structure. This complex structure breaks forms into bidegrees. The Hopf differential of the immersion is the $(2,0)$ part of the second fundamental form of the immersion. It is a global symmetric 2 -form on $S$. Moreover, the Hopf differential is holomorphic if and only if the immersion has constant mean curvature Hop83. Using the above definition, an immersion is isothermic if the Hopf differential is the product of a real valued function and a holomorphic quadratic differential. In this sense, isothermic immersions are a generalization of constant mean curvature immersions.

Remark 2.6. If $f:(S, \mathcal{Q}) \rightarrow(M, g)$ is an isothermic immersion via Definition 2.17 and $z=x+i y$ is an isothermic chart, then $(x, y)$ are principal, isothermal local coordinates. Thus, away from the zeros of $\mathcal{Q}$, an immersion that is isothermic by the above definition is isothermic in the classical sense. The converse, however, holds only locally.

The new definition of isothermic also fails to define isothermic on some totally umbilic surfaces. For example, the unit sphere in $\mathbb{R}^{3}$ is isothermic under the classical definition. However, the sphere has no nontrivial holomorphic quadratic differentials, so it does not admit a polarization.

There are, however, advantages to this new definition. The umbilic points of the immersion are precisely the zeros of the Hopf differential. Definition 2.17 distinguishes between two types of umbilic points. If an umbilic point is caused by a zero in the polarization, then no isothermic coordinates may be found, but umbilics caused by zeros in the real valued function $m$ will admit isothermic coordinates. A torus always admits a nowhere vanishing polarization. By using this polarization, we will find isothermic tori that have umbilic points that arise from the zero locus of the function $m$.

In addition, Definition 2.17 creates a global Calapso potential on the surface. Classically the Calapso potential was defined using a local isothermic coordinate system [Cal03], with the result that different isothermic coordinates could yield different, albeit related, Calapso potentials. Fixing a polarization and taking an isothermic chart fixes a set of local coordinates that make the Calapso potential well defined globally.

Summarizing, Definition 2.17 is not only sufficient, but also advantageous, for the work that concerns us in this article. However, it is too restrictive to be used as a general definition of isothermic.

Remark 2.7. Let $(S, \mathcal{Q})$ be a polarized surface. For any nonzero constant $c \in \mathbb{R}$, consider the polarization $\hat{\mathcal{Q}}=c \mathcal{Q}$. Note that an immersion $f:(S, \mathcal{Q}) \rightarrow(M, g)$ is isothermic via Definition 2.17] if and only if $f:(S, \hat{\mathcal{Q}}) \rightarrow(M, g)$ is also isothermic 
via this definition. The Calapso potentials for the two polarizations are related by

$$
\hat{\Phi}=\frac{\sqrt{|c|}}{c} \Phi .
$$

Similarly, the coordinate $z: U \rightarrow \mathbb{C}$ is an isothermic chart for $\mathcal{Q}$ if and only if $\hat{z}=\sqrt{c} z$ is an isothermic chart for $\hat{\mathcal{Q}}$.

Assumption 2.2. Unless otherwise noted, we assume that any polarization mentioned after this point is nonvanishing.

Remark 2.8. Consider an immersion $f:(S, \mathcal{Q}) \rightarrow(M, g)$. Let $I$ denote its first fundamental form, and $I I^{(2,0)}$, its Hopf differential. Let $r: M \rightarrow \mathbb{R}^{+}$be a smooth function, and replace $g$ by the conformal metric $\hat{g}=r^{2} g$. With this new metric, the fundamental forms of the immersion become $\hat{I}=r^{2} I$ and $\hat{I I}{ }^{(2,0)}=r I I^{(2,0)}$. Thus, the statement that the Hopf differential is a real valued function times the polarization depends only on the conformal class of the metric on $M$, rather than the metric itself. Moreover, using the notation of Definition 2.17, we see that $\hat{\lambda}=\frac{1}{r} \lambda$ and $\hat{m}=r m$. Thus

$$
\hat{\Phi}=2 \hat{m} \hat{\lambda}=2 m \lambda=\Phi .
$$

This equation shows that the Calapso potential also depends only on the conformal class of the metric on $M$. In other words, the definitions of isothermic and the Calapso potential make sense in Möbius space, where we only have a conformal class of metrics. Given a surface immersed in $\mathcal{M}$, take the metric induced by any frame field along the surface, and apply the definitions to this metric.

Let $f:(S, \mathcal{Q}) \rightarrow \mathcal{M}$ be an umbilic free, conformal, isothermic immersion. Let $B$ be a normal frame along $f$, and let $\beta=B^{-1} d B$ be its Maurer-Cartan form. With respect to the metric $I=\left(\beta_{0}^{1}\right)^{2}+\left(\beta_{0}^{2}\right)^{2}$ induced by $B$, the second fundamental form is given by

$$
I I=\beta_{1}^{3} \beta_{0}^{1}+\beta_{2}^{3} \beta_{0}^{2}=\left(\beta_{0}^{1}\right)^{2}-\left(\beta_{0}^{2}\right)^{2} .
$$

Thus, the Hopf differential is given by

$$
I I^{(2,0)}=\frac{1}{2}\left(\beta_{0}^{1}+i \beta_{0}^{2}\right)^{2} .
$$

On the other hand, let $z=x+i y$ be an isothermic chart. Since the immersion is isothermic, the Hopf differential then has the form $I I^{(2,0)}=m d z d z$. Replacing $\mathcal{Q}$ by $-\mathcal{Q}$ if necessary, we may assume that $m>0$. Since $\mathcal{Q}$ is nowhere vanishing, this implies that the Calapso potential $\Phi=e^{u}>0$. In terms of the complex coordinate $z$, we may write $\beta_{0}^{1}+i \beta_{0}^{2}=h d z$, where $h$ is a smooth function. Since $m>0$, the function $h$ is real valued and non-vanishing. If $h>0$, then it follows that $h=e^{u}$. In particular, note that $\beta_{0}^{1}=e^{u} d x$ and $\beta_{0}^{2}=e^{u} d y$. If, on the other hand, $h<0$, then replace $B$ by the other normal frame,

$$
\tilde{B}=\left(B_{0},-B_{1},-B_{2}, B_{3}, B_{4}\right),
$$

and replace $\beta$ by $\tilde{\beta}=\tilde{B}^{-1} d \tilde{B}$. This yields

$$
e^{u}=-h, \quad \tilde{\beta}_{0}^{1}=e^{u} d x, \quad \tilde{\beta}_{0}^{2}=e^{u} d y .
$$

Thus, for any umbilic free, conformal, isothermic immersion $f:(S, \mathcal{Q}) \rightarrow \mathcal{M}$, and any isothermic chart $z=x+i y$ there exists a unique normal frame such that $\beta_{0}^{1}=e^{u} d x$ and $\beta_{0}^{2}=e^{u} d y$, where $e^{u}$ is the Calapso potential. Applying the 
structure equations (7) to this frame, we see that the Maurer-Cartan form of this frame is

$$
\beta=\left(\begin{array}{ccccc}
2 d u & p_{1} e^{u} d x & p_{3} e^{u} d y & 0 & 0 \\
e^{u} d x & 0 & -u_{y} d x+u_{x} d y & -e^{u} d x & p_{1} e^{u} d x \\
e^{u} d y & u_{y} d x-u_{x} d y & 0 & e^{u} d y & p_{3} e^{u} d y \\
0 & e^{u} d x & -e^{u} d y & 0 & 0 \\
0 & e^{u} d x & e^{u} d y & 0 & -2 d u
\end{array}\right) .
$$

Moreover, the equations (7) take the form

$$
\begin{aligned}
q_{1} & =e^{-u} u_{y}, \\
q_{2} & =-e^{-u} u_{x}, \\
\left(p_{1}+p_{3}\right) & =-\left(1+e^{-2 u}\left(u_{x x}+u_{y y}\right)\right), \\
p_{2} & =0 \\
p_{1 y} & =-u_{y}\left(3 p_{1}+p_{3}\right), \\
p_{3 x} & =-u_{x}\left(p_{1}+3 p_{3}\right) .
\end{aligned}
$$

Note, in particular, that $J=\frac{1}{2}\left(p_{1}+p_{3}\right)$ is completely determined by the function $u$. Also note that for an isothermic immersion, the function $M=p_{2}=0$. The next theorem shows that the condition $M=0$ is not only necessary for an isothermic immersion, but also sufficient.

Theorem 2.3 (Mus97]). Let $S$ be a simply connected Riemann surface, and let $f: S \rightarrow \mathcal{M}$ be an umbilic free conformal immersion. Then $M \equiv 0$ if and only if $(S, f)$ is isothermic (in the classical sense).

Remark 2.9. The above theorem shows that the property of being isothermic is a conformal invariant. In other words, it is preserved under the action of the Möbius group.

Theorem 2.4 ([Cal03, Mus97]). Let $(S, \mathcal{Q})$ be a polarized Riemann surface, and let $z=x+i y$ be an isothermic chart. Let $\triangle=\partial_{x x}+\partial_{y y}$ be the Laplacian. Let $f:(S, \mathcal{Q}) \rightarrow \mathcal{M}$ be an umbilic free, isothermic immersion. Then, the Calapso potential $\Phi$ satisfies the Calapso equation

$$
\triangle\left(\Phi^{-1}(\Phi)_{x y}\right)+2\left(\Phi^{2}\right)_{x y}=0 .
$$

Remark 2.10. The Calapso equation is an integrability condition for an isothermic surface. Let $U$ be a simply connected domain in $\mathbb{C}$, and let $e^{u}: U \rightarrow \mathbb{R}^{+}$be any smooth solution of the Calapso equation. Then there exists a 1-parameter family of isothermic immersions $f_{t}: U \rightarrow \mathcal{M}$, each with Calapso potential $e^{u}$ Mus97.

Definition 2.18. Let $f:(S, \mathcal{Q}) \rightarrow \mathcal{M}$ be an umbilic free, conformal, isothermic immersion with Calapso potential $e^{u}>0$. The immersion is called special if the Calapso potential satisfies the second order partial differential equation

$$
\triangle u=s e^{-2 u}-e^{2 u}
$$

where $s \in \mathbb{R}$ is a constant.

Theorem 2.5 (Cal15, CM98). Let $f:(S, \mathcal{Q}) \rightarrow \mathcal{M}$ be an umbilic free, conformal, isothermic immersion. Then $f$ is special if and only if it is locally Möbius equivalent to a constant mean curvature immersion into $M_{c}$ for some $c \in\{-1,0,1\}$. 
Remark 2.11. The actual theorem stated in [CM98] is stronger than the above result. It characterizes the immersions that are locally equivalent to constant mean curvature immersions into each of the three space forms. However, we do not need this information for our purposes.

Definition 2.19. Let $f:(S, \mathcal{Q}) \rightarrow \mathcal{M}$ be an umbilic free isothermic immersion with Calapso potential $\Phi$. Let $z=x+i y$ be an isothermic chart. If $\Phi_{x} \equiv 0$ (or $\left.\Phi_{y} \equiv 0\right)$, then $f$ is called canal. If $\Phi$ is constant, then $f$ is called Dupin

Theorem 2.6 ([Mus97]). Locally, an umbilic free, isothermic, canal surface is Möbius equivalent to either a surface of revolution, a cone, or a cylinder.

\subsection{Transformations of isothermic immersions.}

\subsubsection{The Christoffel transform.}

Definition 2.20. Let $S$ be a surface. Let $f, \hat{f}: S \rightarrow \mathbb{R}^{3}$ be two immersions. The induced map $f(p) \mapsto \hat{f}(p)$ is a Christoffel transform if

1. the map $f(p) \mapsto \hat{f}(p)$ is conformal, and

2. the tangent plane $T_{f(p)}(S)$ is parallel to the tangent plane $T_{\hat{f}(p)}(S)$ for all $p \in S$.

If $f(S)$ and $\hat{f}(S)$ are not congruent, then the Christoffel transform is called nontrivial. A Christoffel transform of an isothermic immersion is also called a dual isothermic immersion.

Theorem 2.7 (Christoffel, [Dar72]). Let $f: S \rightarrow \mathbb{R}^{3}$ be an umbilic free immersion.

1. If $\hat{f}$ is a nontrivial Christoffel transform of $f$, then $f$ is an isothermic immersion.

2. If $f$ is an isothermic immersion, then $f$ admits (local) nontrivial Christoffel transforms.

\subsubsection{The T-transform.}

Definition 2.21. Two isothermic immersions $f, \hat{f}:(S, \mathcal{Q}) \rightarrow \mathcal{M}$ are said to be T-transforms of each other if they have the same Calapso potential [CM98].

The T-transform was first defined by Calapso Cal03. In [Mus95], E. Musso proves that T-transforms are second order conformal deformations 1

Notice that once the Calapso potential has been chosen, the only coefficients of the Maurer-Cartan form (8) that can vary are $p_{1}$ and $p_{3}$. Thus the Maurer-Cartan form of a T-transform of $f$ can differ from the original Maurer-Cartan form only in these components.

\subsubsection{The Darboux transform.}

Definition 2.22. Let $\sigma: S \rightarrow \mathfrak{S}$ be a sphere congruence, and let $f, \hat{f}: S \rightarrow \mathcal{M}$ be envelopes of $\sigma$. If the induced map $f(p) \mapsto \hat{f}(p)$ is conformal and preserves curvature lines, then $\hat{f}$ is called a Darboux transform of $f$.

Theorem 2.8 (Darboux, Dar99]). $\quad$ 1. If $f: S \rightarrow \mathbb{R}^{3}$ admits a Darboux transform $\hat{f}: S \rightarrow \mathbb{R}^{3}$, then $f$ and $\hat{f}$ are isothermic immersions.

\footnotetext{
${ }^{1}$ Two immersions $f, \hat{f}: U \rightarrow \mathcal{M}$ are second order conformal deformations of each other if there is a smooth map $b: U \rightarrow G$ such that $b(p) \cdot \hat{f}$ and $f$ agree up to order 2 at $p$ for each $p \in U$.
} 
2. An umbilic free isothermic immersion $f: S \rightarrow \mathbb{R}^{3}$ of a simply connected surface admits (locally) a 4-parameter family of non-congruent Darboux transforms.

In [MN97, E. Musso and L. Nicolodi use the method of moving frames to construct Darboux transformations of isothermic immersions. We summarize these results below:

Definition 2.23. Let $f: S \rightarrow \mathcal{M}$ be a conformal immersion. Let $B: U \rightarrow G$ be a frame along $f$, and let $\beta=B^{-1} d B$ be its Maurer-Cartan form. The frame $B$ is called a principal frame if

$$
\begin{aligned}
\beta_{0}^{3} & =0, \\
\beta_{1}^{3} \wedge \beta_{0}^{1} & =0=\beta_{2}^{3} \wedge \beta_{0}^{2}, \\
\beta_{0}^{1} \wedge \beta_{0}^{2} & \neq 0 .
\end{aligned}
$$

Suppose $f:(S, \mathcal{Q}) \rightarrow \mathcal{M}$ is isothermic. Let $z=x+i y$ be an isothermic chart. Then a principal frame satisfies

$$
\beta_{0}^{1} \wedge d x=0=\beta_{0}^{2} \wedge d y .
$$

In addition, there exist smooth functions $l_{1}, l_{2}$ such that

$$
\begin{aligned}
\beta_{1}^{2} & =l_{2} \beta_{0}^{1}-l_{1} \beta_{0}^{2}, \\
d\left(\beta_{0}^{0}-l_{1} \beta_{0}^{1}-l_{2} \beta_{0}^{2}\right) & =0 .
\end{aligned}
$$

Principal frames exist along any umbilic free isothermic immersion.

Definition 2.24. A Darboux isothermic frame is a principal frame $B: U \rightarrow G$ along an isothermic immersion $f:(S, \mathcal{Q}) \rightarrow \mathcal{M}$ such that

$$
\begin{aligned}
\left(-\beta_{1}^{0}+i \beta_{2}^{0}\right) \wedge\left(\beta_{0}^{1}+i \beta_{0}^{2}\right) & =0, \\
\beta_{3}^{0} & =0, \\
\beta_{1}^{0} \wedge \beta_{2}^{0} & \neq 0 .
\end{aligned}
$$

Remark 2.12. Let $B: U \rightarrow G$ be a Darboux isothermic frame along an umbilic free isothermic immersion $f:(S, \mathcal{Q}) \rightarrow \mathcal{M}$. Then the matrix

$$
\hat{B}=\left[B_{4},-B_{1}, B_{2}, B_{3}, B_{0}\right]
$$

is a principal frame along $\left[B_{4}\right]$. In particular, if $\hat{\beta}=\hat{B}^{-1} d \hat{B}$ denotes the MaurerCartan form of $\hat{B}$, then the conditions (11) imply that there exists a nowhere zero function $h: U \rightarrow \mathbb{R}$ such that

$$
\hat{\beta}_{0}^{1}=h \beta_{0}^{1}, \quad \hat{\beta}_{0}^{2}=h \beta_{0}^{2}, \quad \hat{\beta}_{1}^{3}=-\beta_{1}^{3}, \quad \hat{\beta}_{2}^{3}=\beta_{2}^{3} .
$$

This shows that $(x, y)$ are principal, isothermal coordinates for $\hat{f}=\left[B_{4}\right]$ on $U$. Thus $\hat{f}$ is another isothermic immersion. Moreover, since a principal frame is a first order frame, both $f$ and $\hat{f}$ envelope the sphere congruence $B_{3}: U \rightarrow \mathfrak{S}$. The resulting correspondence between $f$ and $\hat{f}$ preserves curvature lines and is conformal. Hence $\hat{f}$ is a Darboux transform of $f$. 
Let $f:(S, \mathcal{Q}) \rightarrow \mathcal{M}$ be an isothermic immersion, let $B: U \rightarrow G$ be a principal frame along $f$, and let $t$ be a smooth function on $U$ such that

$$
\frac{d t}{t}=-2\left(\beta_{0}^{0}-l_{1} \beta_{0}^{1}-l_{2} \beta_{0}^{2}\right)
$$

Consider the deformed matrix valued 1-form

$$
\beta_{t}=\left(\begin{array}{ccccc}
\beta_{0}^{0} & \beta_{1}^{0}+t \beta_{0}^{1} & \beta_{2}^{0}-t \beta_{0}^{2} & \beta_{3}^{0} & 0 \\
\beta_{0}^{1} & 0 & -\beta_{1}^{2} & -\beta_{1}^{3} & \beta_{1}^{0}+t \beta_{0}^{1} \\
\beta_{0}^{2} & \beta_{1}^{2} & 0 & -\beta_{2}^{3} & \beta_{2}^{0}-t \beta_{0}^{2} \\
0 & \beta_{1}^{3} & \beta_{2}^{3} & 0 & \beta_{3}^{0} \\
0 & \beta_{0}^{1} & \beta_{0}^{2} & 0 & -\beta_{0}^{0}
\end{array}\right) .
$$

This form satisfies the Maurer-Cartan integrability condition

$$
d \beta_{t}=-\beta_{t} \wedge \beta_{t}
$$

Thus, there exists a map $A: U \rightarrow G$ such that $A^{-1} d A=\beta_{t}$. Let $V_{0} \in L^{5}$ be any constant vector, and let $V=A^{-1} V_{0}$. Then

$$
d V=-\beta_{t} V .
$$

Conversely, if $V$ is a solution to (12), then $d(A V)=0$. This implies that there is a constant vector $V_{0} \in L^{5}$ such that $A V=V_{0}$.

Let the map $V: U \rightarrow \mathcal{L}^{+}$be a solution of equation (12) for a fixed $t$. Write $V={ }^{t}\left(v^{0}, v^{1}, v^{2}, v^{3}, v^{4}\right)$. The conditions $\langle V, V\rangle=0$ and $v^{0}+v^{4}>0$ imply that $v^{4}$ is nonnegative. Away from the zero locus of $v^{4}$, define $g^{+}(V): S \rightarrow G$ by

$$
g^{+}(V)=\left[\begin{array}{ccc}
\left(v^{4}\right)^{-1} & \left(v^{4}\right)^{-1 t} v & v^{0} \\
0 & I_{3} & v \\
0 & 0 & v^{4}
\end{array}\right], v={ }^{t}\left(v^{1}, v^{2}, v^{3}\right)
$$

Lemma 2.2 (Musso, Nicolodi, MN97]). The frame $\mathrm{Bg}^{+}(V)$ is a Darboux isothermic frame.

Let $B$ be the normal frame along an umbilic free isothermic immersion with Maurer-Cartan form (8). This is a principal frame, and the forms $\beta_{1}^{2}$ and $\beta_{0}^{0}$ are given by

$$
\beta_{1}^{2}=u_{y} d x-u_{x} d y, \quad \beta_{0}^{0}=2 d u .
$$

Thus $\beta_{0}^{0}-l_{1} \beta_{0}^{1}-l_{2} \beta_{0}^{2}=d u$, which implies that $t=n e^{-2 u}$ for a real constant $n$. Write $\beta_{t}=\beta_{n}$. Let $V: U \rightarrow \mathcal{L}^{+}$be a solution of (12), and consider the Darboux isothermic frame

$$
\hat{B}=B g^{+}(V): U \rightarrow G
$$

along $f$. Let $d_{n}(f)=\left[\hat{B}_{4}\right]$ denote the resulting Darboux transform of $f$.

Lemma 2.3 (Musso, Nicolodi, MN97]). Let $\Phi$ denote the Calapso potential of $f$. Then the Calapso potential of $d_{n}(f)$ is given by

$$
\Phi_{n}=\frac{v^{3}}{v^{4}} \Phi .
$$

The set $\left\{v^{3}=0\right\}$ is the umbilic locus of $d_{n}(f)$. 


\section{ISOTHERMIC IMMERSIONS WITH SPHERICAL LINES OF CURVATURE}

Let $f:(S, Q) \rightarrow \mathcal{M}$ be an umbilic free isothermic immersion. Let $z=x+i y$ be an isothermic chart on some subset $U$ of $S$, and let

$$
B=\left(B_{0}, B_{1}, B_{2}, B_{3}, B_{4}\right): U \rightarrow G
$$

be the unique normal frame field on $U$ along $f$ with Maurer-Cartan form (8). Suppose that the $y=$ const. curves are of type (2). Let $v(x)=f\left(x, y_{0}\right)$ be a particular line of curvature, let $b$ be the restriction of $B$ to $v$, and let $\theta_{j}^{i}=v^{*} \omega_{j}^{i}$ be the pullback of the Maurer-Cartan forms to the curve. Then

$$
\theta=\left(\begin{array}{ccccc}
2 u_{x} & p_{1} e^{u} & 0 & 0 & 0 \\
e^{u} & 0 & -u_{y} & -e^{u} & p_{1} e^{u} \\
0 & u_{y} & 0 & 0 & 0 \\
0 & e^{u} & 0 & 0 & 0 \\
0 & e^{u} & 0 & 0 & -2 u_{x}
\end{array}\right) d x
$$

To get a second order frame along $v$, let

$$
g=\left[\begin{array}{ccccc}
1 & 0 & e^{-u} u_{y} & 1 & \frac{1}{2}\left(1+e^{-2 u} u_{y}^{2}\right) \\
0 & 1 & 0 & 0 & 0 \\
0 & 0 & 1 & 0 & e^{-u} u_{y} \\
0 & 0 & 0 & 1 & 1 \\
0 & 0 & 0 & 0 & 1
\end{array}\right]
$$

and perform the change of frame $\tilde{b}=b g$. The frame $\tilde{b}$ has Maurer-Cartan form

$$
\tilde{\theta}=\left(\begin{array}{ccccc}
2 u_{x} & e^{u}\left(p_{1}-\frac{1}{2}\left(1+e^{-2 u} u_{y}^{2}\right)\right) & e^{-u}\left(u_{x} u_{y}+u_{x y}\right) & 2 u_{x} & 0 \\
e^{u} & 0 & 0 & 0 & \tilde{\theta}_{1}^{0} \\
0 & 0 & 0 & 0 & \tilde{\theta}_{2}^{0} \\
0 & 0 & 0 & 0 & 2 u_{x} \\
0 & e^{u} & 0 & 0 & -2 u_{x}
\end{array}\right) d x .
$$

By hypothesis, $v(x)$ is a curve of type $(2)$, so $u_{x} \not \equiv 0$. To get a third order frame along the curve, let

$$
\begin{aligned}
r & =\left(e^{-4 u}\left(u_{x y}+u_{x} u_{y}\right)^{2}+4 e^{-2 u} u_{x}^{2}\right)^{-1 / 4} \\
c & =r^{2}\left(e^{-2 u}\left(u_{x y}+u_{x} u_{y}\right)\right. \\
s & =r^{2}\left(2 e^{-u} u_{x}\right)
\end{aligned}
$$

and set

$$
\tilde{g}=\left[\begin{array}{ccccc}
r^{-1} & 0 & 0 & 0 & 0 \\
0 & 1 & 0 & 0 & 0 \\
0 & 0 & c & -s & 0 \\
0 & 0 & s & c & 0 \\
0 & 0 & 0 & 0 & r
\end{array}\right]
$$

Perform the change of frame given by $\bar{b}=\tilde{b} \tilde{g}$. The form

$$
\bar{\theta}_{2}^{3}=2 r^{4} e^{-2 u}\left(u_{x}^{3} u_{y}+u_{x x} u_{x y}-u_{x} u_{x x y}\right) d x .
$$

Since $v$ is spherical if and only if $\bar{\theta}_{2}^{3}=0$, we see that $v$ is spherical if and only if

$$
u_{x}^{3} u_{y}+u_{x x} u_{x y}-u_{x} u_{x x y}=0
$$


along $v$. Note that if $v$ had been of type $(1)$, then $u_{x} \equiv 0$. In this case, the above differential equation is trivially satisfied. By doing the analagous computation for the $x=$ const. curves, we arrive at the following:

Theorem 3.1. Let $f:(S, Q) \rightarrow \mathcal{M}$ be a conformal, umbilic free, isothermic immersion. Let $z=x+i y$ be an isothermic chart, and let $e^{u}$ be the Calapso potential. Then the $x=$ const. curves are spherical if and only if

$$
u_{x} u_{y}^{3}+u_{y y} u_{x y}-u_{y} u_{x y y}=0
$$

and the $y=$ const. curves are spherical if and only if

$$
u_{x}^{3} u_{y}+u_{x x} u_{x y}-u_{x} u_{x x y}=0 .
$$

Corollary 3.1. Let $f:(S, Q) \rightarrow \mathcal{M}$ be an umbilic free isothermic immersion with spherical lines of curvature in one or two systems. Any T-transform of $f$ preserves the property of spherical lines of curvature.

Corollary 3.2. An umbilic free isothermic canal surface has spherical lines of curvature in both systems. One of these systems consists entirely of circles.

Corollary 3.3. Let $f: S \rightarrow \mathbb{R}^{3}$ be an umbilic free constant mean curvature immersion with spherical lines of curvature in one or two systems. A Christoffel transform of $f$ preserves the property of spherical lines of curvature.

Proof. Without loss of generality, we may assume that $f$ has constant mean curvature $H \equiv 1$. Since $f$ has constant mean curvature, the Hopf differential $I I^{(2,0)}$ is a global, holomorphic quadratic differential. Thus we may chose $\mathcal{Q}=2 I I^{(2,0)}$ as a polarization of $S$. If $z=x+i y$ is an isothermic chart for this polarization, then the Hopf differential of $f$ is $I I^{(2,0)}=\frac{1}{2} d z d z$. Writing the first fundamental form as $I=e^{2 u} d z d \bar{z}$, we then see that the Calapso potential of $f$ is $\Phi=e^{-u}$.

From Cal03], we know that a dual of an isothermic immersion with metric $I$ and Hopf form $I I^{(2,0)}$ as above has metric $d \tilde{s}^{2}=e^{-2 u} d z d \bar{z}$ and Hopf differential

$\tilde{I I}^{(2,0)}=\frac{1}{2} d z d z$. Thus, a dual $\tilde{f}$ has Calapso potential $\tilde{\Phi}=e^{u}$. But if $-u$ satifies (13) and/or (14), then so does $u$.

\section{NON-SPECIAL, NON-CANAL ISOTHERMIC TORI WITH TWO SYSTEMS OF SPHERICAL LINES OF CURVATURE}

This section contains a thorough analysis of an example from [MN97]. We begin by summarizing this example below:

Example 4.1 (The Darboux transforms of Dupin tori, [MN97]). The Calapso potential of a Dupin immersion is constant. Without loss of generality, assume that it is identically one. The structure equations (9) imply that $p_{1}, p_{3}$ are constants such that $1+p_{1}+p_{3}=0$. For an arbitrary constant $m \in \mathbb{R}$, set

$$
e^{u}=1, \quad p_{1}=m, p_{3}=-(1+m) \text {. }
$$

The normal frame $B$ of a Dupin surface then has Maurer-Cartan form

$$
\beta=\left(\begin{array}{ccccc}
0 & m d x & -(1+m) d y & 0 & 0 \\
d x & 0 & 0 & -d x & m d x \\
d y & 0 & 0 & d y & -(1+m) d y \\
0 & d x & -d y & 0 & 0 \\
0 & d x & d y & 0 & 0
\end{array}\right)
$$


Let $-3 / 2<m<1 / 2$, and set $\zeta=\sqrt{1-2 m}$ and $\eta=\sqrt{2 m+3}$. For these values of $m$, the Dupin surface must be a torus of revolution with normal frame

$$
\begin{aligned}
B_{0}= & \frac{2+\eta \sin (\zeta x)}{\sqrt{2} \zeta \eta} \epsilon_{0}+\frac{\cos (\zeta x)}{\zeta} \epsilon_{1}+\frac{\cos (\eta y)}{\eta} \epsilon_{2} \\
& +\frac{\sin (\eta y)}{\eta} \epsilon_{3}+\frac{2-\eta \sin (\zeta x)}{\sqrt{2} \zeta \eta} \epsilon_{4}, \\
B_{1}= & \frac{\cos (\zeta x)}{\sqrt{2}} \epsilon_{0}-\sin (\zeta x) \epsilon_{1}-\frac{\cos (\zeta x)}{\sqrt{2}} \epsilon_{4}, \\
B_{2}= & -\sin (\eta y) \epsilon_{2}+\cos (\eta y) \epsilon_{3}, \\
B_{3}= & \frac{-(2 m+1)-\eta \sin (\zeta x)}{\sqrt{2} \zeta \eta} \epsilon_{0}-\frac{\cos (\zeta x)}{\zeta} \epsilon_{1}+\frac{\cos (\eta y)}{\eta} \epsilon_{2} \\
& +\frac{\sin (\eta y)}{\eta} \epsilon_{3}+\frac{-(2 m+1)+\eta \sin (\zeta x)}{\sqrt{2} \zeta \eta} \epsilon_{4}, \\
B_{4}= & \frac{1+m \eta \sin (\zeta x)}{\sqrt{2} \eta \zeta} \epsilon_{0}+\frac{m \cos (\zeta x)}{\zeta} \epsilon_{1}-\frac{(1+m) \cos (\eta y)}{\eta} \epsilon_{2} \\
& -\frac{(1+m) \sin (\eta y)}{\eta} \epsilon_{3}+\frac{1-m \eta \sin (\zeta x)}{\sqrt{2} \eta \zeta} \epsilon_{4} .
\end{aligned}
$$

Note that $B=\left[B_{0}, B_{1}, B_{2}, B_{3}, B_{4}\right]$ is doubly periodic with $x$-period $2 \pi / \sqrt{1-2 m}$ and $y$-period $2 \pi / \sqrt{2 m+3}$. These are the periods of the given Dupin torus.

Perform a Darboux transform $\hat{f}=d_{n}(f)$. Setting $k=m+n$, the linear system (12) takes the form

$$
\begin{aligned}
d v^{0} & =-k v^{1} d x+(1+k) v^{2} d y \\
d v^{1} & =\left(-v^{0}+v^{3}-k v^{4}\right) d x \\
d v^{2} & =\left(-v^{0}-v^{3}+(1+k) v^{4}\right) d y \\
d v^{3} & =-v^{1} d x+v^{2} d y \\
d v^{4} & =-v^{1} d x-v^{2} d y
\end{aligned}
$$

If $n$ is chosen so that $-3 / 2<k<1 / 2$, then this system has solution

$$
\begin{aligned}
& v^{0}=-k u^{1}+(1+k) u^{2}+\alpha^{0} \\
& v^{1}=d u^{1} / d x \\
& v^{2}=d u^{2} / d y \\
& v^{3}=-u^{1}+u^{2}+\alpha^{3} \\
& v^{4}=-u^{1}-u^{2}+\alpha^{4}
\end{aligned}
$$

where

$$
\begin{aligned}
& u^{1}=c_{1} \cos \sqrt{1-2 k} x+c_{2} \sin \sqrt{1-2 k} x+\frac{\left(-\alpha^{0}+\alpha^{3}-k \alpha^{4}\right)}{1-2 k}, \\
& u^{2}=l_{1} \cos \sqrt{2 k+3} y+l_{2} \sin \sqrt{2 k+3} y+\frac{\left(-\alpha^{0}-\alpha^{3}+(1+k) \alpha^{4}\right)}{2 k+3} .
\end{aligned}
$$

Here $c_{1}, c_{2}, l_{1}, l_{2}, \alpha^{0}, \alpha^{3}, \alpha^{4}$ are constants that must be chosen to satisfy $\langle V, V\rangle=0$. Note that $V$ is doubly periodic with $x$-period $2 \pi / \sqrt{1-2 k}$ and $y$-period $2 \pi / \sqrt{2 k+3}$.

The Darboux transform

$$
\hat{f}=B V: \mathbb{R}^{2}-\left\{v^{4}=0\right\} \rightarrow \mathcal{M}
$$


of the original Dupin immersion is given by

$$
\hat{f}=v^{0} B_{0}+v^{1} B_{1}+v^{2} B_{2}+v^{3} B_{3}+v^{4} B_{4} .
$$

Notice that the domain of $\hat{f}$ is a subset of the universal cover of the original torus.

We now show that explicit examples of non-special, non-canal isothermic tori with spherical lines of curvature in two systems arise from the Darboux transforms of Dupin tori computed in the preceding example. This involves a thorough analysis of these transforms. In the following, we always assume that

$$
-\frac{3}{2}<k, m<\frac{1}{2}
$$

Our analysis is broken up into three main parts. Recall that a Darboux transform of a Dupin torus actually takes place on some subset of the universal cover of the torus. We study the vector $V$ to determine when the Darboux transform is defined on all of $\mathbb{R}^{2}$, and to determine when the Darboux transform has umbilic points. Second, we show that a generic Darboux transform of a Dupin torus is neither canal nor special. We also show that it has spherical lines of curvature in two systems. Finally, although the vector $V$ and the matrix $B$ are each doubly periodic, their periods are not equal, so the resulting map $B V$ is not doubly periodic in most cases. Thus, we must determine conditions that make the Darboux transform into a doubly periodic immersion.

There are eight unknown constants in (17) and (18). In picking values for these constants, first pick $k$, followed by $c_{1}, c_{2}, l_{1}, l_{2}$. These five constants determine $V$ : Set

$$
\begin{aligned}
& \tilde{u}^{1}=c_{1} \cos (\sqrt{1-2 k} x)+c_{2} \sin (\sqrt{1-2 k} x), \\
& \tilde{u}^{2}=l_{1} \cos (\sqrt{2 k+3} y)+l_{2} \sin (\sqrt{2 k+3} y) .
\end{aligned}
$$

Then the components of $V$ become

$$
\begin{aligned}
& v^{0}=-k \tilde{u}^{1}+(1+k) \tilde{u}^{2}+\frac{2 \alpha^{0}-(1+2 k) \alpha^{3}+\alpha^{4}}{(1-2 k)(2 k+3)}, \\
& v^{1}=\frac{d \tilde{u}^{1}}{d x}, \\
& v^{2}=\frac{d \tilde{u}^{2}}{d y}, \\
& v^{3}=-\tilde{u}^{1}+\tilde{u}^{2}+\frac{(1+2 k)\left(2 \alpha^{0}-(1+2 k) \alpha^{3}+\alpha^{4}\right)}{(1-2 k)(2 k+3)}, \\
& v^{4}=-\tilde{u}^{1}-\tilde{u}^{2}+\frac{2\left(2 \alpha^{0}-(1+2 k) \alpha^{3}+\alpha^{4}\right)}{(1-2 k)(2 k+3)} .
\end{aligned}
$$

Note the similarity in the constant terms of $v^{0}, v^{3}$, and $v^{4}$. The condition $\langle V, V\rangle=0$ is given by

$$
\begin{aligned}
\left(2 \alpha^{0}-(1+2 k) \alpha^{3}+\alpha^{4}\right)^{2}= & (1-2 k)^{2}(3+2 k)\left(c_{1}^{2}+c_{2}^{2}\right) \\
& +(1-2 k)(3+2 k)^{2}\left(l_{1}^{2}+l_{2}^{2}\right) .
\end{aligned}
$$


Since the quantities $(1-2 k)$ and $(3+2 k)$ are positive on $(-3 / 2,1 / 2)$, equation (20) is always consistent. This equation also shows that the quantity $\left(2 \alpha^{0}-(1+\right.$ $\left.2 k) \alpha^{3}+\alpha^{4}\right)^{2}$ is determined by $k, c_{i}, l_{i}$. The condition $v^{0}+v^{4}>0$ then forces $2 \alpha^{0}-(1+2 k) \alpha^{3}+\alpha^{4}>0$. Looking at (19), we see that $V$ is then completely determined by $k, c_{i}, l_{i}$.

The next lemma shows that, given $k$, we may always choose $c_{i}, l_{i}$ so that $v^{4}$ is nonvanishing. When $v^{4}$ is nowhere zero, the Darboux transform is defined on the entire universal cover of the torus.

Lemma 4.1. The component $v^{4}$ of $V$ is nowhere zero unless

$$
(1-2 k) \sqrt{c_{1}^{2}+c_{2}^{2}}=(2 k+3) \sqrt{l_{1}^{2}+l_{2}^{2}} .
$$

Proof. The condition that $v^{4}$ never vanish is

$$
2 \alpha^{0}-(1+2 k) \alpha^{3}+\alpha^{4}>\frac{(1-2 k)(2 k+3)}{2}\left(\sqrt{c_{1}^{2}+c_{2}^{2}}+\sqrt{l_{1}^{2}+l_{2}^{2}}\right) .
$$

If we square both sides and use 20, this becomes

$$
\left((1-2 k) \sqrt{c_{1}^{2}+c_{2}^{2}}-(2 k+3) \sqrt{l_{1}^{2}+l_{2}^{2}}\right)^{2}>0 .
$$

This inequality will hold unless

$$
(1-2 k) \sqrt{c_{1}^{2}+c_{2}^{2}}=(2 k+3) \sqrt{l_{1}^{2}+l_{2}^{2}} .
$$

By Lemma 2.3 the umbilic locus of the Darboux transform is the zero locus of the function $v^{3}$. The next lemma investigates this zero locus.

Lemma 4.2. If $k \in[-1,0]$, then the function $v^{3}(x, y)$ will have zeros for all values of $c_{1}, c_{2}, l_{1}, l_{2}$. If $k \in\left(-\frac{3}{2},-1\right)$ or $k \in\left(0, \frac{1}{2}\right)$, then there are values of $c_{1}, c_{2}, l_{1}, l_{2}$ that make $v^{3}(x, y)$ nowhere zero, and there are values for which $v^{3}(x, y)$ has zeros. In some cases these zeros are isolated, while in other cases the zero locus consists of curves.

Proof. The condition that $v^{3}$ be nowhere zero is

$$
|1+2 k|\left(2 \alpha^{0}-(1+2 k) \alpha^{3}+\alpha^{4}\right)>(1-2 k)(2 k+3)\left(\sqrt{c_{1}^{2}+c_{2}^{2}}+\sqrt{l_{1}^{2}+l_{2}^{2}}\right) .
$$

Squaring both sides and using (20) yields

$$
\begin{array}{r}
-(1+k)(1-2 k)^{2}\left(c_{1}^{2}+c_{2}^{2}\right)+k(2 k+3)^{2}\left(l_{1}^{2}+l_{2}^{2}\right) \\
-(1-2 k)(2 k+3) \sqrt{c_{1}^{2}+c_{2}^{2}} \sqrt{l_{1}^{2}+l_{2}^{2}}>0 .
\end{array}
$$

Note that if $k \in[-1,0]$, then the left-hand side of the above inequality will be nonpositive, so this inequality will never hold.

Now suppose that $k \in\left(0, \frac{1}{2}\right)$. Set $u=\sqrt{c_{1}^{2}+c_{2}^{2}}$ and $v=\sqrt{l_{1}^{2}+l_{2}^{2}}$. After replacing the inequality in (21) with equality, we get

$$
A(k) u^{2}+B(k) u v+C(k) v^{2}=0 .
$$

For fixed $k$, this is the equation of a degenerate conic section in $(u, v)$. The discriminant is

$$
\left(B^{2}-4 A C\right)(k)=(1-2 k)^{2}(2 k+3)^{2}(1+2 k)^{2},
$$


which is strictly positive on $k \in\left(0, \frac{1}{2}\right)$. Thus, the conic is a degenerate hyperbola. We will show that this hyperbola consists of two lines through the origin, one of which passes through the interior of the first quadrant.

Change coordinates by rotating the $u, v$ axes through the angle $\alpha$ defined by

$$
\alpha=\frac{1}{2} \operatorname{arccot}\left(\frac{A-C}{B}\right) .
$$

The function $(A-C) / B$ is increasing as a function of $k$ on $\left[0, \frac{1}{2}\right)$, with range $\left[\frac{1}{3}, \infty\right)$. Thus, $\alpha$ decreases as a function of $k$ from $\frac{1}{2} \operatorname{arccot}\left(\frac{1}{3}\right)$ to 0 on $\left[0, \frac{1}{2}\right]$.

If $\tilde{u}, \tilde{v}$ represent the new coordinates after rotation, then the conic equation takes the form

$$
\tilde{A}(k) \tilde{u}^{2}+\tilde{C}(k) \tilde{v}^{2}=0
$$

where

$$
\begin{aligned}
& \tilde{A}=A \cos ^{2} \alpha+B \cos \alpha \sin \alpha+C \sin ^{2} \alpha \\
& \tilde{C}=A \sin ^{2} \alpha-B \cos \alpha \sin \alpha+C \cos ^{2} \alpha .
\end{aligned}
$$

The function $\tilde{A}(k)$ increases on $\left[0, \frac{1}{2}\right]$ from $-\frac{1}{2}-\sqrt{\frac{5}{2}}$ to 0 , while the function $\tilde{C}(k)$ increases from $-\frac{1}{2}+\sqrt{\frac{5}{2}}$ to 8 . Thus we see that the degenerate hyperbola is formed by the two lines

$$
\tilde{v}=\sqrt{\frac{-\tilde{A}}{\tilde{C}}} \tilde{u} \text { and } \quad \tilde{v}=-\sqrt{\frac{-\tilde{A}}{\tilde{C}}} \tilde{u} \text {. }
$$

Let

$$
\beta=\arctan \left(\sqrt{\frac{-\tilde{A}}{\tilde{C}}}\right)
$$

Note that $\beta$ is the angle of inclination that the first line in 22 makes with the $\tilde{u}$ axis. The function $\beta(k)$ decreases on $[0,1 / 2]$ from

$$
\arctan \left(\sqrt{\frac{\sqrt{10}+1}{\sqrt{10}-1}}\right)
$$

to 0 .

The angle of inclination of the line with the $u$ axis is given by $\alpha+\beta$. The function $(\alpha+\beta)(k)$ is decreasing on $[0,1 / 2]$, and elementary trigonometry shows that $\lim _{k \rightarrow 0}(\alpha+\beta)=\pi / 2$ and $\lim _{k \rightarrow 1 / 2}(\alpha+\beta)=0$. Thus, this line always passes through the interior of the first quadrant. On one side of this line, there will be a neighborhood in the interior of the first quadrant where (21) holds, while on the other side of this line, there will be a neighborhood where the inequality in (21) is reversed. On the line itself,

$$
\begin{aligned}
&-(1+k)(1-2 k)^{2}\left(c_{1}^{2}+c_{2}^{2}\right)+k(2 k+3)^{2}\left(l_{1}^{2}+l_{2}^{2}\right) \\
&-(1-2 k)(2 k+3) \sqrt{c_{1}^{2}+c_{2}^{2}} \sqrt{l_{1}^{2}+l_{2}^{2}}=0 .
\end{aligned}
$$

In this case, the zeros of $v^{3}$ will be isolated points provided that neither $\tilde{u}^{1}$ nor $\tilde{u}^{2}$ is constant. To see this, look at the expression for $v^{3}$ in (19). When (23) holds, 
then

$$
\frac{|2 k+1|\left(2 \alpha^{0}-(1+2 k) \alpha^{3}+\alpha^{4}\right)}{(1-2 k)(2 k+3)}=-\min _{(x, y) \in \mathbb{R}^{2}}\left(-\tilde{u}^{1}+\tilde{u}^{2}\right)=\max _{(x, y) \in \mathbb{R}^{2}}\left(-\tilde{u}^{1}+\tilde{u}^{2}\right) .
$$

If neither $\tilde{u}^{1}$ nor $\tilde{u}^{2}$ is constant, the minima and maxima of $-\tilde{u}^{1}+\tilde{u}^{2}$ are isolated points, so this shows that the zeros of $v^{3}$ are isolated. On the other hand, when the inequality in (21) is reversed,

$$
\frac{|2 k+1|\left(2 \alpha^{0}-(1+2 k) \alpha^{3}+\alpha^{4}\right)}{(1-2 k)(2 k+3)}<-\min _{(x, y) \in \mathbb{R}^{2}}\left(-\tilde{u}^{1}+\tilde{u}^{2}\right)=\max _{(x, y) \in \mathbb{R}^{2}}\left(-\tilde{u}^{1}+\tilde{u}^{2}\right),
$$

so the zero locus of $v^{3}$ will contain curves.

The case $k \in\left(-\frac{3}{2},-1\right)$ is similar.

Corollary 4.1. Let the map $f: \mathbb{R}^{2} \rightarrow \mathcal{M}$ be a Dupin torus with Maurer-Cartan form (15). If $k \in[-1,0]$, then any Darboux transform $d_{k-m}(f)$ of $f$ will have curves of umbilic points given by the zero locus of $v^{3}$. If $k \in\left(-\frac{3}{2},-1\right)$ or $k \in\left(0, \frac{1}{2}\right)$, then some Darboux transforms $d_{k-m}(f)$ will have curves of umbilics, some will have isolated umbilics, and some will be umbilic free. Umbilic points are again given by the zero locus of $v^{3}$.

This completes the analysis of the vector $V$. Next we examine the properties of a generic Darboux transform of a Dupin torus.

Lemma 4.3. A Darboux transform of a Dupin torus is canal if and only if either $c_{1}=0=c_{2}$ or $l_{1}=0=l_{2}$.

Proof. A Darboux transform will be canal if and only if its Calapso potential, $v^{3} / v^{4}$, is a function of one variable; that is, if and only if either $\frac{d u^{1}}{d x} \equiv 0$ or $\frac{d u^{2}}{d y} \equiv 0$.

Lemma 4.4. A Darboux transform of a Dupin torus is not special.

Proof. It suffices to show that, away from umbilic points, the Calapso potential of a Darboux transform does not satisfy (10). Away from umbilic points, we may assume without loss of generality that $v^{3}>0$. The Calapso potential may then be written $\Phi=e^{u}$, where

$$
u=\ln v^{3}-\ln v^{4}
$$

By (16),

$$
\triangle u=-1-\frac{\left(v^{1}\right)^{2}+\left(v^{2}\right)^{2}}{\left(v^{3}\right)^{2}}+\frac{\left(v^{1}\right)^{2}+\left(v^{2}\right)^{2}-2 v^{0} v^{4}}{\left(v^{4}\right)^{2}}+(1+2 k) \frac{v^{4}}{v^{3}}
$$

Suppose that

$$
\triangle u=s e^{-2 u}-e^{2 u}
$$

for some constant $s \in \mathbb{R}$. These two expressions for $\triangle u$, along with the equation $\langle V, V\rangle=0$, imply

$$
s v^{4}=-2 v^{0}+(1+2 k) v^{3} .
$$

Using (17), we obtain

$$
(1-s) u^{1}+(1-s) u^{2}=-2 \alpha^{0}+(1+2 k) \alpha^{3}-s \alpha^{4} .
$$


If $s \neq 1$, then (24) implies that

$$
\begin{aligned}
& u^{1}=\text { const. } \\
& u^{2}=\text { const. }
\end{aligned}
$$

This occurs if and only if $c_{1}, c_{2}, l_{1}$, and $l_{2}$ are all zero; that is, if and only if $V$ is constant. In particular, (17) implies that $v^{1} \equiv 0 \equiv v^{2}$. Since the derivatives of $v^{1}, v^{2}$ must then vanish, 16 implies

$$
\begin{aligned}
-v^{0}+v^{3}-k v^{4} & =0, \\
-v^{0}-v^{3}+(1+k) v^{4} & =0 .
\end{aligned}
$$

Adding these two equations yields $v^{4}=2 v^{0}$, and back substituting then implies $v^{3}=(1+2 k) v^{0}$. The condition $\langle V, V\rangle=0$ then forces $V$ to be identically zero, which is impossible.

If, on the other hand, $s=1$, then

$$
-2 \alpha^{0}+(1+2 k) \alpha^{3}-\alpha^{4}=0 .
$$

Then (20) implies that both $u_{1}$ and $u_{2}$ are constant, which, as we just demonstrated, is impossible.

Lemma 4.5. A Darboux transform of a Dupin immersion is an immersion if and only if $v^{4}$ is never zero and $k \neq m$.

Proof. Recall that a Darboux transform of a Dupin torus is given by

$$
\hat{f}=v^{0} B_{0}+v^{1} B_{1}+v^{2} B_{2}+v^{3} B_{3}+v^{4} B_{4}
$$

Using (15) and (16), we compute

$$
\begin{aligned}
& \frac{d \hat{f}}{d x}=(m-k) v^{1} B_{0}+(m-k) v^{4} B_{1}, \\
& \frac{d \hat{f}}{d y}=(k-m) v^{2} B_{0}+(k-m) v^{4} B_{2} .
\end{aligned}
$$

These two vectors will span a 2-dimensional subspace of the tangent space of $\mathcal{M}$ if and only if $\hat{f}, \hat{B}_{x}, \hat{B}_{y}$ are linearly independent; that is, if and only if $v^{4}$ is never zero and $k \neq m$.

The next lemma proves that a Darboux transform of a Dupin immersion has spherical curvature lines in both systems. It is possible to do this using Theorem 3.1. However, on the one hand, this is a computational mess, and on the other hand, some of the Darboux transforms are not umbilic free. Instead, we use the more direct approach of computing third order frames along the curvature lines.

Lemma 4.6. A Darboux transform of a Dupin immersion has spherical curvature lines in both systems.

Proof. Recall that the frame $\hat{B}=B g^{+}(v)$ is a Darboux isothermic frame along the Dupin immersion, whose fourth column vector is a Darboux transform of the original surface. Consider the frame

$$
\tilde{B}:=\left(\hat{B}_{4},-\hat{B}_{1}, \hat{B}_{2}, \hat{B}_{3}, \hat{B}_{0}\right) .
$$


This is a principal frame along the immersion $\left[B_{4}\right]$. It has Maurer-Cartan form

$$
\left(\begin{array}{ccccc}
0 & -\frac{d x}{v^{4}} & \frac{d y}{v^{4}} & 0 & 0 \\
v^{4} n d x & 0 & -\frac{v^{2}}{v^{4}} d x+\frac{v^{1}}{v^{4}} d y & \left(1-\frac{v^{3}}{v^{4}}\right) d x & -\frac{d x}{v^{4}} \\
v^{4} n d y & \frac{v^{2}}{v^{4}} d x-\frac{v^{1}}{v^{4}} d y & 0 & \left(1+\frac{v^{3}}{v^{4}}\right) d y & \frac{d y}{v^{4}} \\
0 & -\left(1-\frac{v^{3}}{v^{4}}\right) d x & -\left(1+\frac{v^{3}}{v^{4}}\right) d y & 0 & 0 \\
0 & v^{4} n d x & v^{4} n d y & 0 & 0
\end{array}\right)
$$

Take the frame $b=\tilde{B}(\cdot, y)$ along a $y=$ const. curve. Perform the change of frame $\tilde{b}=b g$, where

$$
g=\left[\begin{array}{ccccc}
1 & 0 & \frac{v^{2}}{\left(v^{4}\right)^{2} n} & -\frac{v^{4}-v^{3}}{\left(v^{4}\right)^{2} n} & \frac{1}{2} \frac{\left(v^{2}\right)^{2}+\left(v^{4}-v^{3}\right)^{2}}{\left(v^{4}\right)^{4} n^{2}} \\
0 & 1 & 0 & 0 & 0 \\
0 & 0 & 1 & 0 & \frac{v^{2}}{\left(v^{4}\right)^{2} n} \\
0 & 0 & 0 & 1 & -\frac{v^{4}-v^{3}}{\left(v^{4}\right)^{2} n} \\
0 & 0 & 0 & 0 & 1
\end{array}\right] .
$$

The frame $\tilde{b}$ is a second order frame along the curve. Let $\tilde{\beta}$ denote the MaurerCartan form of $\tilde{b}$. Then

$$
\begin{aligned}
\tilde{\beta}_{2}^{0} & =\frac{2 v^{1} v^{2}}{n^{2}\left(v^{4}\right)^{4}} \tilde{\beta}_{0}^{1}, \\
\tilde{\beta}_{3}^{0} & =\frac{2 v^{1}\left(v^{3}-v^{4}\right)}{n^{2}\left(v^{4}\right)^{4}} \tilde{\beta}_{0}^{1} .
\end{aligned}
$$

Note that both $v^{2}$ and $\left(v^{3}-v^{4}\right)$ are functions of $y$ alone, so they are constant along the curvature line. If both are zero, then the curve is a circle, and hence spherical. If at least one of $v^{2}$ or $\left(v^{3}-v^{4}\right)$ is nonzero, then let

$$
\begin{gathered}
r=\frac{|n|\left(v^{4}\right)^{2}}{\left(4\left(v^{1}\right)^{2}\left(\left(v^{2}\right)^{2}+\left(v^{3}-v^{4}\right)^{2}\right)\right)^{1 / 4}}, \\
c=r^{2} \frac{2 v^{1} v^{2}}{n^{2}\left(v^{4}\right)^{4}} \\
s=r^{2} \frac{2 v^{1}\left(v^{3}-v^{4}\right)}{n^{2}\left(v^{4}\right)^{4}},
\end{gathered}
$$

and set

$$
\tilde{g}=\left[\begin{array}{ccccc}
r^{-1} & 0 & 0 & 0 & 0 \\
0 & 1 & 0 & 0 & 0 \\
0 & 0 & c & -s & 0 \\
0 & 0 & s & c & 0 \\
0 & 0 & 0 & 0 & r
\end{array}\right]
$$

Perform the change of frame $\hat{b}=\tilde{b} \tilde{g}$.

Away from the zeros of $v^{1}(x)$, this gives a third order frame along the curve for which $\hat{\beta}_{2}^{3}=0$. Thus, between any two zeros of $v^{1}$, the curve lies in a sphere. In particular, the curve lies in the sphere

$$
\hat{b}_{3}=\frac{v^{1}}{\left|v^{1}\right|} \frac{1}{\sqrt{\left(v^{2}\right)^{2}+\left(v^{3}-v^{4}\right)^{2}}}\left(\left(v^{4}-v^{3}\right) b_{2}+v^{2} b_{3}\right) .
$$


This shows that the vector

$$
\frac{1}{\sqrt{\left(v^{2}\right)^{2}+\left(v^{3}-v^{4}\right)^{2}}}\left(\left(v^{4}-v^{3}\right) b_{2}+v^{2} b_{3}\right),
$$

which is continuous on the entire curve, is constant between the zeros of $v^{1}$. If $v^{1} \equiv 0$, then the curve is of type (1), and hence spherical. If $v^{1} \not \equiv 0$, then the zeros of $v^{1}$ are isolated on the curve, so continuity implies that the above vector is constant along the entire curve. The two vectors

$$
\pm \frac{1}{\sqrt{\left(v^{2}\right)^{2}+\left(v^{3}-v^{4}\right)^{2}}}\left(\left(v^{4}-v^{3}\right) b_{2}+v^{2} b_{3}\right)
$$

define the same sphere with opposite orientations. Hence, the entire curve is spherical.

For the $x=$ const. curves, take the frame $\bar{b}=\tilde{B}(x,$.$) along an x=$ const. curve. An argument analogous to the one above proves that this curve either is a circle, or lies in the sphere

$$
\frac{-v^{2}}{\left|v^{2}\right|} \frac{1}{\sqrt{\left(v^{1}\right)^{2}+\left(v^{3}+v^{4}\right)^{2}}}\left(\left(v^{3}+v^{4}\right) b_{1}+v^{1} b_{3}\right) .
$$

Remark 4.1. The Maurer-Cartan form (25) shows that $(x, y)$ are global principal, isothermal coordinates along the immersion, so we see directly that the immersion is isothermic in the classical sense.

Finally, we turn to double periodicity of the Darboux transform.

Lemma 4.7. A Darboux transform of a Dupin torus is doubly periodic if and only if the numbers

$$
\frac{\sqrt{1-2 k}}{\sqrt{1-2 m}} \quad \text { and } \quad \frac{\sqrt{2 k+3}}{\sqrt{2 m+3}}
$$

are both rational.

Proof. The vector $V$ is periodic in $x$ with period $2 \pi / \sqrt{1-2 k}$ and periodic in $y$ with period $2 \pi / \sqrt{2 k+3}$. The matrix $B$ is periodic in $x$ with period $2 \pi / \sqrt{1-2 m}$ and periodic in $y$ with period $2 \pi / \sqrt{2 m+3}$. The Darboux transform $\hat{f}=B V$ will be doubly periodic if and only if the ratio of the two $x$ periods is rational and the ratio of the two $y$ periods is rational.

Lemma 4.8. The set of $(k, m)$ in $\left(-\frac{3}{2}, \frac{1}{2}\right) \times\left(-\frac{3}{2}, \frac{1}{2}\right)$ with $k \neq m$ that satisfy the system of equations

$$
\frac{\sqrt{1-2 k}}{\sqrt{1-2 m}}=\frac{p}{q}, \quad \frac{\sqrt{2 k+3}}{\sqrt{2 m+3}}=\frac{r}{s},
$$

where $p, q, r, s$ are positive integers satisfying

$$
p \neq q, \quad r \neq s, \quad \frac{p}{q} \neq \frac{r}{s}
$$

is countably infinite. 
Proof. Assume that (26) holds, and solve for $k$ in terms of $p, q, r, s$ :

$$
k(p, q, r, s)=\frac{3 p^{2}\left(s^{2}-r^{2}\right)-r^{2}\left(p^{2}-q^{2}\right)}{2\left(q^{2} r^{2}-s^{2} p^{2}\right)} .
$$

This implies that

$$
m(p, q, r, s)=\frac{2 k(p, q, r, s) q^{2}+p^{2}-q^{2}}{2 p^{2}} .
$$

Since $p, q, r, s$ are all integers, the number $k$, and hence the number $m$, is rational.

Let $a$ be a positive integer, and consider

$$
\begin{gathered}
k(a, a+1, a+1, a)=\frac{1+2 a-2 a^{2}}{2+4 a+4 a^{2}}, \\
m(a, a+1, a+1, a)=\frac{-\left(3+6 a+2 a^{2}\right)}{2+4 a+4 a^{2}} .
\end{gathered}
$$

For $a>0$, elementary calculus arguments show that $k$ is strictly decreasing and lies in $\left(-\frac{1}{2}, \frac{1}{2}\right)$, while $m$ is strictly increasing and lies in $\left(-\frac{3}{2},-\frac{1}{2}\right)$. Thus there are infinitely many distinct pairs $(k, m)$ that satisfy (26).

Lemma 4.9. The non-special isothermic tori given by Darboux transformations of Dupin tori belong to a countably infinite family of conformal classes.

Proof. With notation as in the proof of the last lemma, let $(k, m)$ be a solution of (26). Assume that $\frac{p}{q}$ and $\frac{r}{s}$ are in lowest terms. Then the Darboux transform $\hat{f}=d_{k-m}(f)$ is doubly periodic with $x$-period and $y$-period

$$
\frac{2 q \pi}{\sqrt{1-2 m}} \text { and } \frac{2 s \pi}{\sqrt{2 m+3}}
$$

respectively; that is, $\hat{f}$ descends to an immersion of $\mathbb{R}^{2} / \Lambda$, where $\Lambda$ is the lattice generated by these two periods.

Let $\mathbb{R}^{2} / \Lambda_{1}$ and $\mathbb{R}^{2} / \Lambda_{2}$ be two such tori, defined by $m_{1}=m\left(p_{1}, q_{1}, r_{1}, s_{1}\right)$ and $m_{2}=m\left(p_{2}, q_{2}, r_{2}, s_{2}\right)$ respectively. These tori are in the same conformal class if and only if

$$
\frac{s_{1} \sqrt{1-2 m_{1}}}{q_{1} \sqrt{2 m_{1}+3}}=\frac{s_{2} \sqrt{1-2 m_{2}}}{q_{2} \sqrt{2 m_{2}+3}} .
$$

Consider

$$
\begin{aligned}
k(a, a+1, a+2, a+1) & =\frac{1+3 a-a^{3}}{2(1+a)^{3}}, \\
m(a, a+1, a+2, a+1) & =\frac{-(2+a)}{2(1+a)} .
\end{aligned}
$$

For integers $a>0$, both $k$ and $m$ lie in $\left(-\frac{3}{2}, \frac{1}{2}\right)$, and both $p / q$ and $r / s$ are always in lowest terms. Moreover,

$$
\frac{s \sqrt{1-2 m}}{q \sqrt{2 m+3}}=\frac{\sqrt{3+2 a}}{\sqrt{1+2 a}} .
$$

The right hand side of this equation is strictly decreasing as a function of $a$, so each positive integer $a$ gives rise to a distinct conformal class of tori. 
Lemma 4.10. If two isothermic immersions $f, \hat{f}:(S, Q) \rightarrow \mathcal{M}$ are Möbius equivalent, then they have the same Calapso potential.

Proof. Let $B$ be a first order frame field along $f$. By hypothesis, there exists $A \in G$ such that $\hat{f}=A f$. Then $\hat{B}=A B$ is a first order frame field along $\hat{f}$, and

$$
\beta=B^{*} \omega=(A B)^{*} \omega=\hat{\beta},
$$

where $\omega$ is the Maurer-Cartan form of $G$. In particular, since $B$ and $\hat{B}$ have the same Maurer-Cartan form, Remark 2.8 implies that the Calapso potentials of the two immersions are equal.

Lemma 4.11. The set of non-Möbius-equivalent, non-canal tori given by Darboux transformations of Dupin isothermic tori is uncountable. In particular, let $(k, m)$ be a fixed solution of (26), and let $f, \hat{f}$ be Darboux transforms defined by two solutions of equation (18). If

$$
\left(\hat{c}_{1}, \hat{c}_{2}, \hat{l}_{1}, \hat{l}_{2}\right) \neq w\left(c_{1}, c_{2}, l_{1}, l_{2}\right)
$$

where $w>0$ is a real constant, then $f$ and $\hat{f}$ are not Möbius equivalent.

Proof. If $f$ and $\hat{f}$ are Möbius equivalent, then, by the preceding lemma they have the same Calapso potential; that is,

$$
v^{3} \hat{v}^{4}=v^{4} \hat{v}^{3} .
$$

This implies that

$$
\left(u^{1} \hat{u}^{2}-\hat{u}^{1} u^{2}\right)=a(x)+b(y) .
$$

In particular,

$$
\left(u^{1} \hat{u}^{2}-\hat{u}^{1} u^{2}\right)_{x y}=0
$$

which implies that

$$
\left(\hat{c}_{1}, \hat{c}_{2}, \hat{l_{1}}, \hat{l_{2}}\right)=w\left(c_{1}, c_{2}, l_{1}, l_{2}\right)
$$

for some constant $w \in \mathbb{R}^{*}$. Then

$$
2 \hat{\alpha}^{0}-(1+2 k) \hat{\alpha}^{3}+\hat{\alpha}^{4}=|w|\left(2 \alpha^{0}-(1+2 k) \alpha^{3}+\alpha^{4}\right) .
$$

Substituting all this back into (27), we see that $w$ must be positive.

We summarize our results in the following theorem:

Theorem 4.1. The set $\mathcal{T}$ of isothermic tori given by the Darboux transforms of Example 4.1 contains a countably infinite number of distinct conformal classes. Each conformal class contains an uncountable number of non-Möbius-equivalent tori. The elements of $\mathcal{T}$ have spherical lines of curvature in two systems. They are not special, and a generic element of $\mathcal{T}$ is not canal. Some elements of $\mathcal{T}$ are umbilic free, some have isolated umbilic points, and some have curves of umbilic points distinct from the coordinate curvature lines. 


\section{EXAMPLES AND Figures}

Computer graphics of the tori found in Section 4 can be generated as follows. First choose $k, m, c_{1}, c_{2}, l_{1}$, and $l_{2}$. Then, using Example 4.1 compute the functions $u_{i}, v^{j}, B_{j}$, where $i \in\{1,2\}$, and $j \in\{0,1,2,3,4\}$. Next, simplify the expression

$$
B V=v^{0} B_{0}+v^{1} B_{1}+v^{2} B_{2}+v^{3} B_{3}+v^{4} B_{4},
$$

which gives the components of the desired Darboux transform. Let

$$
{ }^{t}\left[f^{0}, f^{1}, f^{2}, f^{3}, f^{4}\right]
$$

denote the components of $B V$. Using a parametric plot over a fundamental domain of the torus, plot the stereographic projection of this map into $\mathbb{R}^{3}$, which is given by

$$
\sqrt{2}\left(\frac{f^{1}}{f^{0}}, \frac{f^{2}}{f^{0}}, \frac{f^{3}}{f^{0}}\right) .
$$

The resulting image will be the desired torus.

Example 5.1. We choose $k$ and $m$ by using the proof of Lemma 4.8, that is, we pick $p, q, r, s$ so that

$$
k(2,3,2,1)=-1 / 4, \quad m(2,3,2,1)=-19 / 16 .
$$

We then arbitrarily choose

$$
c_{1}=1, \quad c_{2}=0, \quad l_{1}=1, \quad l_{2}=0 .
$$

Using (201), we compute

$$
2 \alpha^{0}-(1+2 k) \alpha^{3}+\alpha^{4}=\sqrt{15}
$$

The $x$-period is $4 \pi / \sqrt{3 / 2}$, and the $y$-period is $4 \pi / \sqrt{5 / 2}$. The conformal class is given by the ratio of the $y$-period and the $x$-period, in this case, $\sqrt{3 / 5}$. Figure 1 shows a picture of this torus.

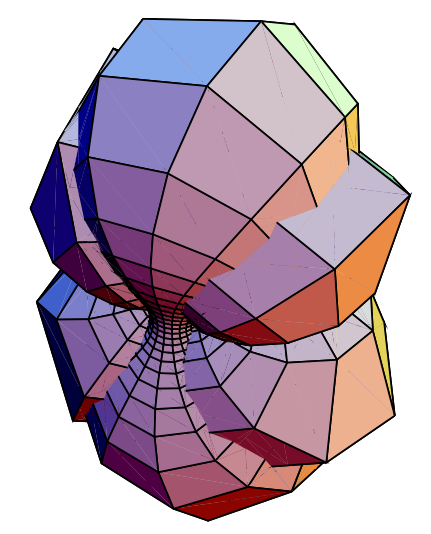

Figure 1. A torus in conformal class $\sqrt{3 / 5}$. 

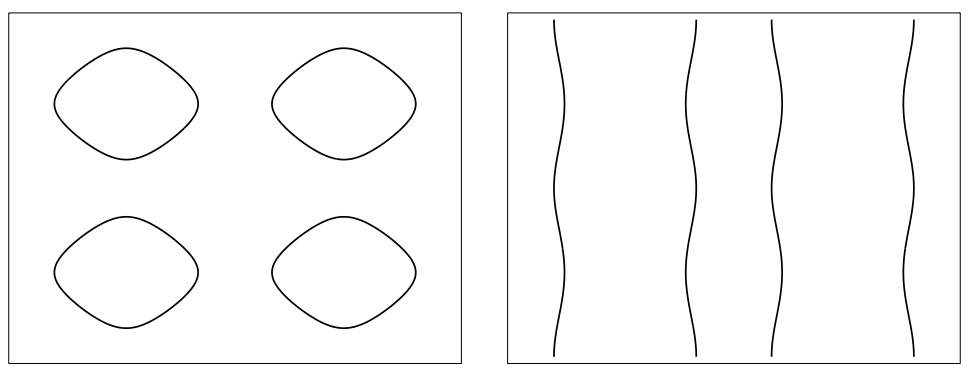

Figure 2. The umbilic loci of the tori in Figures 1 and 3 , respectively, shown in a single fundamental domain.
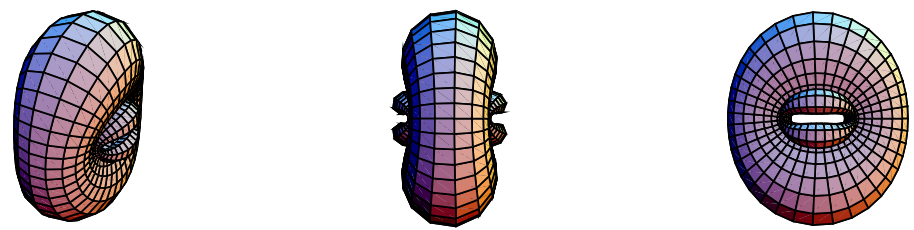

Figure 3. Another torus in conformal class $\sqrt{3 / 5}$ shown from three different viewpoints.

Since $k \in[-1,0]$, the torus generated by these constants will necessarily have umbilic points. The umbilic locus of this torus is shown in Figure 2 In the connected region, the function $v^{3}$ is positive, on the four umbilic curves, it is zero, and in the oval shaped regions, $v^{3}<0$.

Example 5.2. In this example, we create a torus that is not Möbius equivalent to the preceding example, but is still in the same conformal class. Let $k, m$ be as in Example 5.1 but this time choose

$$
c_{1}=7, \quad c_{2}=0, \quad l_{1}=1, \quad l_{2}=0 .
$$

This implies $2 \alpha^{0}-(1+2 k) \alpha^{3}+\alpha^{4}=\sqrt{285}$. By Lemma 4.11, this torus is not Möbius equivalent to the previous example. However since $k$ and $m$ are the same for both tori, they are in the same conformal class. Figure 3 shows a picture of this torus from three different viewpoints.

Again, since $k \in[-1,0]$, this torus has a nonempty umbilic locus, pictured in Figure 2,

Continuing in this manner, one can generate further images. By changing $k$ and $m$, one can produce tori in other conformal classes, though some distinct choices of $k, m$ will yield tori in the same conformal class. Moreover, if $k$ is in the appropriate range, then one can produce tori that are umbilic free by manipulating the $c_{i}$ and $l_{i}$ so that equation 21 is satisfied. 


\section{REFERENCES}

[Abr87] U. Abresch, Constant mean curvature tori in terms of elliptic functions, Journal für die Reine und Angewandte Mathematik 374 (1987), 169-192. MR 88e:53006

[Bob91] A. Bobenko, All constant mean curvature tori in $\mathbb{R}^{3}, S^{3}, \mathbb{H}^{3}$ in terms of theta functions, Math. Ann. 290 (1991), 209-245. MR 92h:53072

[Bry84] R. Bryant, A duality theorem for Willmore surfaces, Journal of Differential Geometry 20 (1984), 23-53. MR 86j:58029

[Cal03] P. Calapso, Sulle superficie a linea di curvatura isoterme, Rend. Circ. Mat. Palermo 17 (1903), 275-286.

[Cal15] P. Calapso, Sulle transformazioni delle superficie isoterme, Annali di Mat. 24 (1915), $11-48$.

[CM98] D. Carfí and E. Musso, T-transformations of Willmore isothermic surfaces, Proceedings of Conference in Messina: Congresso Internazionali di Geometria Differenzialee in onore di Pasquale Calapso (Messina), Oct 1998.

[Dar99] G. Darboux, Sur les surfaces isothermiques, C. R. Acad. Sci. Paris 128 (1899), 12991305.

[Dar72] G. Darboux, Leçons sur la théorie générale des surfaces et les applications géométriques du calcul infinitésimal, vols. II, IV, Chelsea Publishing Company, New York, 1972. MR 53:80 MR 53:82

[Hop83] H. Hopf, Differential geometry in the large, Springer-Verlag, New York, 1983, Lecture Notes in Mathematics, 1000. MR 85b:53001

[KPP98] G. Kamberov, P. Pedit, and U. Pinkall, Bonnet pairs and isothermic surfaces, Duke Mathemathics Journal 92 (1998), 637-644. [MR 99h:53009

[LT81] B. Lawson and R. Tribuzy, On the mean curvature function for compact surfaces, Journal of Differential Geometry 16 (1981), 179-183. MR 83e:53060

[MN97] E. Musso and L. Nicolodi, Bäcklund's theorem for the Calapso equation, Tech. report, CNR, 1997.

[Mus95] E. Musso, Deformazione di superfici nello spazio di Möbius, Rend. Istit. Mat. Univ. Trieste 27 (1995), 25-45. MR 97i:53013

[Mus97] E. Musso, Möbius geometry, Unpublished notes, 1997.

[Nic96] L. Nicolodi, Preliminaries about Möbius geometry, Unpublished notes, 1996.

[PS89] U. Pinkall and I. Sterling, On the classification of constant mean curvature tori, Ann. of Math. 130 (1989), 407-451. MR 91b:53009

[Sul81] R. Sulanke, Submanifolds of the Möbius space, II: Frenet formulas and curves of constant curvature, Math. Nachr. 100 (1981), 235-247. MR 83d:53046

[Tri80] R. Tribuzy, A characterization of tori with constant mean curvature in a space form, Bol. Soc. Brasil. Mat. 11 (1980), 259-274. MR 84d:53066

[Wal87] R. Walter, Explicit examples to the H-problem of Heinz Hopf, Geometriae Dedicata 23 (1987), 187-213. MR 88i:53015a

[Wal89] R. Walter, Constant mean curvature tori with spherical curvature lines in non-euclidean geometry, Manuscripta Mathematica 63 (1989), no. 3, 343-363. MR 90a:53016

[Wen86] H. Wente, Counterexample to a conjecture of H. Hopf, Pacific Jour. of Math. 121 (1986), 193-243. MR 87d:53013

Department of Mathematics, Washington University in St. Louis, Campus Box 1146, One Brookings Drive, St. Louis, Missouri 63130

E-mail address: holly@math.wustl.edu 